\title{
Guía de abordaje y manejo endoscópico de la hemorragia de tubo digestivo bajo
}

\author{
Claudia Isabel Blanco-Vela1, Mario Peláez-Luna ${ }^{2 *}$, Sergio Solana-Sentíes ${ }^{3}$, \\ Clara Luz Martínez-García4, Angélica I. Hernández-Guerrero ${ }^{5}$, Jony Cerna-Cardona ${ }^{6}$, \\ Alejandra Noble-Lugo ${ }^{7}$, Orlando Bada-Yllán ${ }^{8}$, Gerardo Blanco-Velasco ${ }^{9}$, Óscar Víctor Hernández-Mondragón ${ }^{9}$, \\ Claudia Martínez-Camacho ${ }^{10}$, Yolanda Zamorano-Orozco ${ }^{10}$, Ángel Mario Zárate-Guzmán ${ }^{11}$, María Elena \\ López-Acosta ${ }^{12}$, Xochiquetzal Sánchez-Chávez ${ }^{13}$, María del Carmen Manzano-Robleda ${ }^{5}$, Ángel Andrés \\ Reyes-Dorantes ${ }^{14}$, José Luis Rocha-Ramírez ${ }^{15}$, Juan Carlos Sánchez-Robles ${ }^{16}$, Rodrigo Soto-Solís ${ }^{17}$ y \\ Gerardo Alfredo Montejo-Ruiz ${ }^{18}$
}

\begin{abstract}
${ }^{1}$ Servicio de Gastroenterología, Hospital San Ángel Inn Universidad, Hospital Central Militar; ${ }^{2}$ División de Investigación, Facultad de Medicina UNAM; Departamento de Gastroenterología, Instituto Nacional de Ciencias Médicas y Nutrición Salvador Zubirán; ${ }^{3}$ Servicio de Endoscopia, Hospital Central Ignacio Morones Prieto; ${ }^{4}$ Servicio Endoscopia, Hospital San Ángel Inn Universidad; ${ }^{5}$ Servicio de Endoscopia, Instituto Nacional de Cancerología; ${ }^{6}$ Servicio de Endoscopia, Hospital Juárez de México; ${ }^{7}$ Departamento de Enseñanza, Hospital Español; ${ }^{8}$ División de Cirugía General y Endoscópica, Hospital General Dr. Manuel Gea González; ${ }^{9}$ Servicio de Endoscopia, Hospital de Especialidades Centro Médico Nacional Siglo XXI, IMSS; ${ }^{10}$ Servicio de Endoscopia, Hospital General Regional N. ${ }^{\circ} 1$ Dr. Carlos MacGregor Sánchez-Navarro; ${ }^{11}$ Unidad de Endoscopia, Hospital General de México Dr. Eduardo Liceaga. Ciudad de México; ${ }^{12}$ Servicio de Endoscopia, Hospital Ángeles Lomas, Estado de México; ${ }^{13}$ Servicio de Endoscopia, Hospital San Ángel Inn Patriotismo; ${ }^{14}$ Asociación Mexicana de Endoscopia Gastrointestinal; ${ }^{15}$ Cirugía de Colon y Recto, Hospital San Ángel Inn Universidad; ${ }^{16}$ Servicio de Colon y Recto, Hospital Central Militar; ${ }^{17}$ Servicio de Endoscopia, Centro Médico Nacional 20 de Noviembre; ${ }^{18}$ Servicio de Radiología e Imagen Molecular, Centro Médico ABC, Campus Santa Fe. Ciudad de México, México
\end{abstract}

\section{Resumen}

La hemorragia de tubo digestivo bajo (HTDB) se define como la originada distal a la válvula ileocecal; su presentación clínica puede ser con hematoquecia, rectorragia o melena. La evaluación inicial debe incluir historia clínica rápida, examen físico y estudios de laboratorio para determinar la severidad de la hemorragia, etiología y posible localización de esta. El primer paso en el tratamiento de la hemorragia aguda de tubo digestivo bajo es evaluar el estado hemodinámico y la reanimación intensiva antes de realizar colonoscopia, que se mantiene como el método diagnóstico y terapéutico de elección. La información específica sobre su tratamiento es escasa por lo que el manejo suele adaptarse de las recomendaciones sobre el manejo de la hemorragia de tubo digestivo alto. También, a pesar de la existencia de diversas guías internacionales, su aplicación y disponibilidad en Mexico es limitada, por lo que la Asociación Mexicana de Endoscopia Gastrointestinal convocó a un grupo de gastroenterólogos, endoscopistas y coloproctólogos para revisar los avances en el manejo de la HTDB. La presente guía se enfoca en el origen, abordaje, tratamiento y seguimiento de la HTDB de acuerdo con directrices internacionales y a la disponibilidad de recursos humanos y tecnológicos en nuestro país. Para su realización se utilizó el

\section{Correspondencia:}

*Mario Peláez-Luna

División de Investigación, Facultad de Medicina, UNAM

Departamento de Gastroenterología, INCMNSZ

Vasco de Quiroga, 15

Col. Belisario Domínguez, Sección XVI, Del. Tlalpan Fecha de recepción: 14-12-2018

C.P. 14080, Ciudad de México, México

Fecha de recepción: 14-12-2018

Disponible en internet: 05-02-2019

E-mail: mariopl@prodigy.net.mx

DOI: 10.24875/END.M19000002

Endoscopia. 2019;31:4-29

www.endoscopia-ameg.com

0188-9893/@ 2018. Asociación Mexicana de Endoscopia Gastrointestinal, publicado por Permanyer México SA de CV, todos los derechos reservados. 
método Delphi. La evaluación de la evidencia y grado recomendación se realizó utilizando el sistema GRADE (Grading of Recommendations, Assessment, Development and Evaluation) modificado.

Palabras clave: Hemorragia tubo digestivo. Colonoscopia. Hematoquecia. Rectorragia. Guías de manejo clínico.

\title{
Guide to endoscopic management and treatment of low digestive tract hemorrhage
}

\begin{abstract}
Lower gastrointestinal bleeding (LGIB) localizes distal to the ileocecal valve. It presents either as hematochezia, rectal bleeding and melena in some cases. Initial approach must include a complete and detailed clinical and physical exam, laboratory exams in order to determine the severity and source of the hemorrhage. The first step in the treatment of LGIB is to asses and stabilize the patient hemodynamic status and prepare for colonoscopy, which remains the best diagnostic and therapeutic tool. Although LGIB accounts for a significant number of emergencies an hospital admissions, management information usually comes from data obtained and adjusted for upper gastrointestinal bleeding studies. Despite several guidelines have been published, its availability and applicability in our country is limited, therefore, the Asociación Mexicana de Endoscopia Gastrointestinal summoned a group of gastroenterologists, endoscopists and colon and rectum surgeons in order to review, discuss and grade current data on LGIB in order to develop management recommendations for $L G I B$. The resultant guideline focuses on the etiology, management and follow up of patients presenting LGIB in accordance to international standards and resources available in our country. Guidelines were developed using the Delphi method and evidence was grades using the modified Grading of Recommendations, Assessment, Development and Evaluation System).
\end{abstract}

Key words: Gastrointestinal bleeding. Colonoscopy. Hematochezia. Rectal bleeding. Clinical guidelines.

\section{Introducción}

La hemorragia de tubo digestivo bajo (HTDB) se define como la originada distal a la válvula ileocecal; su presentación clínica puede ser con hematoquecia, rectorragia o melena. La HTDB representa el $20-25 \%$ de todos los casos de hemorragia digestiva y ocurre con mayor frecuencia en hombres que en mujeres. En México no se cuenta con información precisa sobre su incidencia. El primer paso en el manejo de la hemorragia aguda de tubo digestivo bajo (HATDB) es evaluar el estado hemodinámico y la reanimación intensiva antes de realizar colonoscopia, que se mantiene como el método diagnóstico y terapéutico de elección. La información específica sobre su tratamiento es escasa por lo que el manejo suele adaptarse de las recomendaciones sobre el manejo de la hemorragia de tubo digestivo alto.

A pesar de la existencia de diversas guías internacionales, su aplicación y disponibilidad en México es limitada, por lo que en marzo del 2018 la Asociación Mexicana de Endoscopia Gastrointestinal (AMEG) convocó a un grupo de gastroenterólogos, endoscopistas y coloproctólogos para revisar los avances en el tratamiento de la HTDB, con la finalidad de evaluar de manera crítica la evidencia científica y proponer estrategias de diagnóstico y tratamiento mediante un consenso, y establecer guías de manejo. La presente guía se enfoca en el origen, abordaje, tratamiento y seguimiento de la HTDB de acuerdo con las directrices internacionales y a la disponibilidad de recursos humanos y tecnológicos en nuestro país, y se basa en la evidencia científica disponible en la actualidad.

\section{Métodos}

Para preparar y realizar la elaboración de guías consensuadas se utilizó el método Delphi'. Se crearon cuatro mesas de trabajo y a cada una se le asignó un tema específico que representa cada una de las secciones de la presente guía. Cuatro de los participantes realizaron una búsqueda en la base de datos PubMed de artículos publicados a manera de resumen o artículo completo en los idiomas inglés y español. Se dio preferencia a revisiones sistemáticas, metaanálisis, guías de práctica clínica, consensos, ensayos clínicos controlados y de cohortes, aunque no se limitó a este tipo de publicaciones.

Una vez concluida la búsqueda se elaboraron enunciados relacionados con el tema de cada mesa y fueron enviados a cada coordinador de mesa junto con la bibliografía seleccionada. Cada coordinador asignó aleatoriamente uno o más enunciados a cada 
Tabla 1. Evaluación GRADE

\begin{tabular}{|l|l|}
\hline Niveles de calidad & Definición \\
\hline Alto & Alta confianza en la coincidencia entre el efecto real y el estimado \\
\hline Moderado & $\begin{array}{l}\text { Moderada confianza en la estimación del efecto. Hay posibilidad de que el efecto real esté alejado del efecto } \\
\text { estimado }\end{array}$ \\
\hline Bajo & Confianza limitada en la estimación del efecto. El efecto real puede estar lejos del estimado \\
\hline Muy bajo & Poca confianza en el efecto estimado. El efecto verdadero muy probablemente sea diferente del estimado \\
\hline
\end{tabular}

GRADE: Grading of Recommendations, Assessment, Development and Evaluation.

participante, quien calificó la evidencia que los apoya. Para evaluar la calidad de la evidencia publicada y seleccionar los artículos más relevantes sin importar si los resultados eran positivos o negativos se utilizó el sistema GRADE (Grading of Recommendations, Assessment, Development and Evaluation) modificado².

Con la finalidad de homogeneizar la forma de evaluación y que cada participante conociera el método, se les pidió que realizaran el curso en línea gratuito Up to Date del sistema GRADE y se les envió un enlace electrónico a dicho curso. Este sistema tiene el objetivo de mejorar la evaluación de la calidad de la evidencia y la fuerza con la que esta se recomienda. En él la graduación de la calidad no se basa en el tipo de estudio (diseño y metodología), sino que se consideran también los desenlaces (pertinencia, relevancia, etc.), así como las preguntas elaboradas para estudiar dicho desenlace. De forma que incluso cuando la evidencia de mayor calidad se origina en revisiones sistemáticas, ensayos clínicos, etc., el sistema GRADE clasifica la calidad basándose en el diseño y métodos utilizados para evaluar los desenlaces preseleccionados o responder una pregunta tipo PICO (Población o Pacientes, Intervención o Indicador, Control o Comparación y $\mathrm{O}$ de desenlace por su traducción del inglés Outcome) elaborada con anterioridad y con fines específicos. Esto permite que pueda ser considerada evidencia de menor calidad (p. ej., casos y controles, estudios transversales, series de casos) $)^{3}$.

La calidad de la evidencia puede ser «alta» cuando la seguridad o confianza en los desenlaces 0 efectos estimados no puede o no podría ser modificada por investigaciones subsecuentes. Se considera «moderada» cuando investigaciones posteriores podrían modificar los efectos o desenlaces y, por lo tanto, nuestra confianza en la información actual disminuye. La calidad es «baja» cuando es muy probable que la información actual se modifique con estudios ulteriores y «muy baja» cuando los desenlaces o efectos en estudio conocidos en la actualidad son extremadamente inciertos (Tabla 1).

La calidad y fuerza de recomendación (débil o fuerte) se expresan como letras mayúsculas de la « $A$ » a la "D», que indican la calidad de evidencia (A para muy alta calidad y $\mathrm{D}$ para muy baja calidad), y números, que indican la fuerza de recomendación a favor o en contra (1 para fuerte y 2 para débil) (Tabla 2).

Estos enunciados y su calificación fueron presentados por los coordinadores a los miembros de sus mesas, donde se revisó su contenido y redacción y se efectuaron modificaciones en conjunto, además de discutir la pertinencia o necesidad de eliminar o agregar otros enunciados. Se votó "acuerdo» o «desacuerdo" para cada enunciado. Cuando el acuerdo fue mayor al $75 \%$ se concluyó que el enunciado permanecería sin modificaciones, y cuando el acuerdo fue menor al $75 \%$ se realizaron modificaciones en contenido y redacción, sometiéndose nuevamente a votación, y así sucesivamente hasta llegar a un acuerdo mayor al $75 \%$.

Aquellos enunciados con desacuerdo mayor del $75 \%$, repetidos o redundantes, fueron eliminados del proceso. Posteriormente a la reunión cada coordinador y los miembros de cada mesa desarrollaron comentarios y consideraciones finales a cada enunciado, además de revisar y determinar el nivel y calidad de evidencia que lo apoya, así como la fortaleza de la recomendación, basándose en el sistema GRADE modificado antes mencionado (Tabla 2).

Esta guía puede y deberá ser revisada y editada periódicamente de acuerdo con la necesidad de incorporar cambios y avances tecnológicos, así como información nueva y relevante. Las recomendaciones 
Tabla 2. Recomendaciones GRADE

\begin{tabular}{|c|c|c|c|}
\hline $\begin{array}{l}\text { Grado de } \\
\text { recomendación }\end{array}$ & Claridad del riesgo/beneficio & Calidad de la evidencia de apoyo & Implicaciones \\
\hline $\begin{array}{l}1 \mathrm{~A} \\
\text { Recomendación fuerte/ } \\
\text { Calidad de evidencia } \\
\text { alta }\end{array}$ & $\begin{array}{l}\text { Los beneficios claramente } \\
\text { superan los riesgos o } \\
\text { viceversa }\end{array}$ & $\begin{array}{l}\text { Evidencia consistente proveniente de } \\
\text { ensayos controlados y aleatorizados o } \\
\text { bien evidencia convincente de alguna } \\
\text { otra forma. Estudios posteriores } \\
\text { difícilmente impactarán en nuestra } \\
\text { confianza y cambiarán el estimado de } \\
\text { los riesgos y beneficios }\end{array}$ & $\begin{array}{l}\text { Las recomendaciones } \\
\text { fuertes pueden aplicarse a } \\
\text { todos los casos en casi } \\
\text { todas las circunstancias }\end{array}$ \\
\hline $\begin{array}{l}\text { 1B } \\
\text { Recomendación fuerte/ } \\
\text { Calidad de evidencia } \\
\text { moderada }\end{array}$ & $\begin{array}{l}\text { Los beneficios claramente } \\
\text { superan los riesgos o } \\
\text { viceversa. Existe incertidumbre } \\
\text { en las estimaciones de riesgo } \\
\text { y beneficio }\end{array}$ & $\begin{array}{l}\text { Evidencia de ensayos clínicos } \\
\text { controlados con limitaciones } \\
\text { importantes (resultados inconsistentes, } \\
\text { fallas metodológicas o imprecisiones) o } \\
\text { evidencia muy fuerte de estudios con } \\
\text { otro diseño. Investigaciones futuras (si } \\
\text { se realizan) pueden impactar en la } \\
\text { confianza y modificar los beneficios y } \\
\text { riesgos estimados }\end{array}$ & $\begin{array}{l}\text { Recomendación fuerte que } \\
\text { podría aplicarse a todos los } \\
\text { casos en casi todas las } \\
\text { circunstancias a menos que } \\
\text { existan una alternativa clara } \\
\text { congruente y racional }\end{array}$ \\
\hline $\begin{array}{l}\text { 1C } \\
\text { Recomendación fuerte/ } \\
\text { Calidad de evidencia } \\
\text { baja }\end{array}$ & $\begin{array}{l}\text { Los beneficios aparentemente } \\
\text { superan los riesgos o } \\
\text { viceversa }\end{array}$ & $\begin{array}{l}\text { Evidencia de estudios observacionales, } \\
\text { experiencia clínica no sistematizada o } \\
\text { de ensayos clínicos controlados con } \\
\text { errores serios. Cualquier estimación de } \\
\text { riesgo o beneficio es incierta }\end{array}$ & $\begin{array}{l}\text { La recomendación fuerte } \\
\text { puede aplicarse a casi todos } \\
\text { los pacientes, pero la } \\
\text { evidencia que la apoya es } \\
\text { de baja calidad }\end{array}$ \\
\hline $\begin{array}{l}2 \mathrm{~A} \\
\text { Recomendación débil } \\
\text { con evidencia de } \\
\text { calidad alta }\end{array}$ & $\begin{array}{l}\text { Los beneficios están } \\
\text { equilibrados o son similares a } \\
\text { los riesgos }\end{array}$ & $\begin{array}{l}\text { Evidencia consistente proveniente de } \\
\text { ensayos controlados y aleatorizados o } \\
\text { bien evidencia convincente de alguna } \\
\text { otra forma. Estudios posteriores } \\
\text { difícilmente impactarán en nuestra } \\
\text { confianza y cambiarán la estimación de } \\
\text { los riesgos y beneficios }\end{array}$ & $\begin{array}{l}\text { La recomendación débil } \\
\text { implica que la mejor acción } \\
\text { o estrategia puede cambiar } \\
\text { dependiendo de las } \\
\text { circunstancias clínicas y } \\
\text { sociales de cada caso }\end{array}$ \\
\hline $\begin{array}{l}\text { 2B } \\
\text { Recomendación débil/ } \\
\text { Calidad de evidencia } \\
\text { moderada }\end{array}$ & $\begin{array}{l}\text { Los beneficios están } \\
\text { equilibrados o son similares a } \\
\text { los riesgos. Existe } \\
\text { incertidumbre en las } \\
\text { estimaciones de riesgo y } \\
\text { beneficio }\end{array}$ & $\begin{array}{l}\text { Evidencia de ensayos clínicos } \\
\text { controlados con limitaciones } \\
\text { importantes (resultados inconsistentes, } \\
\text { fallas metodológicas o imprecisiones) o } \\
\text { evidencia muy fuerte de estudios con } \\
\text { otro diseño. Investigaciones futuras (si } \\
\text { se realizan) pueden impactar en la } \\
\text { confianza y modificar los beneficios y } \\
\text { riesgos estimados }\end{array}$ & $\begin{array}{l}\text { Recomendación débil. } \\
\text { Tratamientos alternativos } \\
\text { pueden considerarse } \\
\text { mejores en algunos } \\
\text { pacientes y bajo ciertas } \\
\text { circunstancias }\end{array}$ \\
\hline $\begin{array}{l}2 \mathrm{C} \\
\text { Recomendación débil/ } \\
\text { Calidad de evidencia } \\
\text { baja }\end{array}$ & $\begin{array}{l}\text { Incertidumbre en las } \\
\text { estimaciones de riesgo y } \\
\text { beneficio. Los beneficios } \\
\text { posiblemente están } \\
\text { balanceados o son similares a } \\
\text { los riesgos }\end{array}$ & $\begin{array}{l}\text { Evidencia de estudios observacionales, } \\
\text { experiencia clínica no sistematizada o } \\
\text { de ensayos clínicos controlados con } \\
\text { errores serios. Cualquier estimación de } \\
\text { riesgo o beneficio es incierta }\end{array}$ & $\begin{array}{l}\text { Recomendación muy débil. } \\
\text { Otras alternativas pudieran } \\
\text { ser igualmente razonables }\end{array}$ \\
\hline
\end{tabular}

GRADE: Grading of Recommendations, Assessment, Development and Evaluation.

presentadas y la fuerza de estas se basan en la revisión de la evidencia científica disponible en el momento de su elaboración, así como en la discusión y evaluación de los riesgos y beneficios hecha por los participantes de la guía. Es importante recordar que esta, como todas las guías, presenta información que pretende ayudar a los involucrados en el cuidado de pacientes y no deben considerarse reglas ni estándares de cuidado inmodificables, puesto que las decisiones clínicas deben individualizarse y suelen ser resultado de un análisis detallado de la situación clínica del paciente y los recursos disponibles que pudieran 0 no estar en acuerdo con este documento. 


\section{Generalidades de la hemorragia de tubo digestivo bajo}

\section{Definición}

LA HEMORRAGIA DE TUBO DIGESTIVO BAJO SE DEFINE COMO LA ORIGINADA DISTAL A LA VÁLVULA ILEOCECAL; SU PRESENTACIÓN CLÍNICA PUEDE SER CON HEMATOQUECIA, RECTORRAGIA O MELENA

\section{Recomendación fuerte, calidad de evidencia baja (GRADE 1C)}

La HTDB se define históricamente como la pérdida anormal de sangre originada en algún punto entre el ángulo de Treitz y el ano; sin embargo, a partir del desarrollo de la cápsula endoscópica y el enteroscopio se ha modificado su definición y abordaje diagnóstico. Estas y otras tecnologías son la causa de la introducción del concepto de hemorragia de intestino medio, la cual se origina desde el ámpula de Vater hasta el íleon terminal, por lo que en la actualidad la HTDB es aquella que ocurre entre el ciego o válvula ileocecal hasta el ano ${ }^{4}$.

La HTDB puede presentarse de forma aguda, crónica y recurrente. La HATDB se define como aquella de reciente inicio (arbitrariamente, tres días) y es autolimitada. La HATDB grave es aquella que provoca inestabilidad hemodinámica en el paciente, causa anemia aguda, requiere de hemotransfusión y en ocasiones tratamiento en Unidades de Cuidado Intensivo (UCI). La manifestación clínica clásica de la HATDB es la hematoquecia (sangre marrón o roja fresca a través del ano). Cuando proviene del lado derecho del colon puede presentarse como melena y en algunas situaciones como hematoquecia.

La hemorragia crónica es aquella que ocasiona pérdida de sangre en forma intermitente o muy lenta durante varios días (más de tres), incluso aquella que el paciente no nota (hemorragia oculta). La hemorragia recurrente es aquella que reincide en su presentación una vez que ya había cedido la sintomatología inicial (24 h) y es de las mismas características ${ }^{5-7}$. Se define recurrencia temprana a aquella que ocurre cuando el paciente se encuentra hospitalizado y tardía cuando lo hace hasta 30 días después de su egreso.

\section{Epidemiología}

LA HEMORRAGIA AGUDA DE TUBO DIGESTIVO BAJO REPRESENTA HASTA EL 25\% DE LAS HEMORRAGIAS GASTROINTESTINALES. LA FRECUENCIA ETIOLÓGICA VARÍA DE ACUERDO CON EL GRUPO ETARIO

\section{Recomendación fuerte, calidad de evidencia baja (GRADE 1C)}

La HTDB representa el $20-25 \%$ de todos los casos de hemorragia digestiva y ocurre con mayor frecuencia en hombres que en mujeres ${ }^{5-10}$. En México no se cuenta con información precisa sobre la incidencia; en EE.UU. se ha reportado que ocurren 20-30 casos por cada 100,000 individuos, mientras que en los Países Bajos la incidencia es de 9/100,000 por año. La frecuencia se ha incrementado debido al aumento en la edad media poblacional, asociado a un mayor número de comorbilidades, mayor uso de aspirina y otros fármacos antitrombóticos ${ }^{5,7,9}$.

La incidencia y el riesgo aumentan con la edad; la edad de presentación más frecuente es entre 63 y 77 años, y se ha observado un riesgo 200 veces mayor entre los 20 y 80 años. Los pacientes con HTDB se encuentran en un, ${ }^{6}$. En comparación con la hemorragia de tubo digestivo alto (HTDA), la HTDB se presenta con menor frecuencia asociada a estado de choque (19 vs. $35 \%$ ) y los requerimientos transfusionales son menores (36 vs. $64 \%$ ). Aproximadamente el $80-85 \%$ de los episodios de HTDB ceden espontáneamente. La tasa de mortalidad general se ubica en el 2-4\%. La etiología ese modifica con la edad ${ }^{9}$, la enfermedad diverticular es la causa más común en pacientes mayores de 40 años, en los cuales constituye la causa del $17-50 \%$ de los casos de HTDB, seguida de angiodisplasias, colitis, pólipos, cáncer colorrectal, enfermedad anorrectal y hemorragia pospolipectomía ${ }^{5-13}$ (Tablas 3 y 4).

\section{Evaluación inicial y estratificación de riesgo en hemorragia aguda de tubo digestivo bajo}

LA EVALUACIÓN INICIAL DEL PACIENTE CON HEMORRAGIA

DE TUBO DIGESTIVO BAJO DEBE INCLUIR HISTORIA

CLÍNICA RÁPIDA, EXAMEN FÍSICO Y ESTUDIOS DE LABORATORIO PARA DETERMINAR LA SEVERIDAD DE LA HEMORRAGIA, LA ETIOLOGÍA Y SU POSIBLE LOCALIZACIÓN

\section{Recomendación fuerte, calidad de evidencia baja (GRADE 1C)}

La severidad de las manifestaciones clínicas de la HATDB depende de la velocidad, el volumen y la localización de la hemorragia7. La presentación más frecuente es la hematoquecia, presente hasta en el $85 \%$ de los casos, seguida de melena con coágulos rojos en el $14 \%$; la melena aislada se observa en una minoría ${ }^{14}$. Si bien la hematoquecia es una manifestación común de la HTDB, no es exclusiva de ella. Después de evaluar factores clínicos que pudieran diferenciar los sitios de hemorragia, Sittichanbuncha, et al..$^{15}$ encontraron 
Tabla 3. Causas de hemorragia de tubo digestivo bajo

\begin{tabular}{|l|c|}
\hline Causas & Porcentaje (\%) \\
\hline Diverticulosis & $30-65$ \\
\hline Colitis isquémica & $5-20$ \\
\hline Hemorroides & $5-20$ \\
\hline Pólipos colorrectales/neoplasias & $2-15$ \\
\hline Angioectasias & $5-10$ \\
\hline Hemorragia pospolipectomía & $2-7$ \\
\hline Ell & $3-5$ \\
\hline Colitis infecciosa & $2-5$ \\
\hline Síndrome de úlcera solitaria & $0-5$ \\
\hline Varices colorrectales & $0-3$ \\
\hline Proctopatía por radiación & $0-2$ \\
\hline Colopatía por AlNE & $0-2$ \\
\hline Lesión de Dieulafoy & Raro \\
\hline
\end{tabular}

AINE: antiinflamatorio no esteroideo; Ell: enfermedad inflamatoria intestinal. Adaptada de Gralnek, et al., 2017\%.

que en 1,854 pacientes con hemorragia de tubo digestivo (HTD) aguda, 76 presentaron hematoquecia; después de una evaluación completa, el $57 \%$ fueron categorizados como HTDB y el $39 \%$ como HTDA. El antecedente de consumo de alcohol, cirrosis o dolor abdominal fue infrecuente en el grupo de HTDB en comparación con el de HTDA. Solo tres factores se asociaron con HTDA en pacientes con hematoquecia: la presión arterial sistólica baja, el nivel de hematocrito disminuido y la relación BUN/Cr elevada.

En otro estudio ${ }^{16}$ se compararon las variables de laboratorio entre grupos con hemorragia aguda de tubo digestivo alto y HATDB $(n=3,296$ y $n=1,520$, respectivamente). La hemoglobina $(\mathrm{Hb})(\mathrm{p}<0.05)$, las proteínas totales $(p<0.001)$ y la deshidrogenasa láctica $(p<0.01)$ fueron significativamente más bajos en el grupo de HTDA en comparación con el de HTDB. El BUN fue el parámetro más fuertemente asociado con HTDA, con un punto de corte $\geq 21 \mathrm{mg} / \mathrm{dl}$ asociado a una especificidad diagnóstica del $93 \%$.

Diversos reportes epidemiológicos indican que la incidencia de HTDA ha disminuido mientras que la de HTDB está en aumento ${ }^{12,17,18}$. El riesgo de recurrencia de esta última ha sido difícil de evaluar, sin embargo, un estudio retrospectivo de 342 pacientes hospitalizados por HATDB encontró que la edad mayor a 65 años (HR: 1.7; $p<0.5$ ), y el uso de antiinflamatorios no esteroideos (AINE) (HR: $2 ; p<0.01)$ y antiplaquetarios (HR: $1.8 ; p<0.5)$ son factores independientes de recurrencia de $\mathrm{HTDB}^{10}$.

\section{LA ESTRATIFICACIÓN DE RIESGO (ALTO O BAJO) PERMITE PREDECIR MORTALIDAD, RIESGO DE RECURRENCIA DE LA HEMORRAGIA, NECESIDAD DE INTERVENCIÓN ENDOSCÓPICA URGENTE E INGRESO A CUIDADOS INTENSIVOS}

\section{Recomendación fuerte, calidad de evidencia mo- derada (GRADE 1B)}

A diferencia de lo observado en la HTDA, los factores pronósticos de recurrencia de hemorragia y mortalidad en la HTDB no han sido claramente establecidos. Los estudios de estratificación de riesgo son escasos y limitados.

Strate, et al..$^{19}$ recolectaron datos sobre 24 aspectos clínicos durante las primeras cuatro horas de evaluación hospitalaria de HATDB en 252 pacientes consecutivos, de los que el $49 \%$ presentó un cuadro grave. El desenlace primario que predecir fue hemorragia grave, definida como hemorragia continua dentro de las primeras $24 \mathrm{~h}$ de hospitalización (transfusión $\geq 2$ unidades de sangre y/o disminución del hematocrito $\geq 20 \%$ ) y/o hemorragia recurrente después de $24 \mathrm{~h}$ de estabilidad (transfusiones adicionales, disminución adicional del hematocrito de $\geq 20 \%$, o readmisión por HATDB durante la semana posterior al alta). Los predictores independientes de hemorragia grave fueron: frecuencia cardíaca $\geq 100 / \mathrm{min}$ (razón de momios [RM]: 3.67; intervalo de confianza del 95\% [IC 95\%]: 1.78-7.57), presión arterial sistólica $\leq 115 \mathrm{mmHg}$ (RM: 3.45; IC 95\%: 1.54-7.72), síncope (RM: 2.82; IC 95\%: 1.06-7.46); examen abdominal normal (RM: 2.43; IC 95\%: 1.22-4.85); hemorragia por el recto durante las primeras cuatro horas de evaluación (RM: 2.32; IC 95\%: 1.284.20); uso de aspirina (OR: 2.07; IC 95\%: 1.12-3.82), y más de dos comorbilidades.

Para validar estas observaciones los mismos autores analizaron estos siete predictores de HATDB grave en una cohorte prospectiva de 275 pacientes $^{20}$. Los pacientes se estratificaron en tres grupos de riesgo de acuerdo con la regla de predicción desarrollada anteriormente: bajo (sin factores de riesgo), moderado (1-3 factores de riesgo) y alto ( $>3$ factores de riesgo). El riesgo de hemorragia severa en cada categoría fue similar en la cohorte de validación y en la de derivación: riesgo bajo 6 vs. $9 \%$, riesgo moderado 43 vs. $43 \%$ y riesgo alto 79 vs. $84 \%$ respectivamente. El área bajo la curva fue de 0.754 para la cohorte de validación y de 0.761 para la cohorte de derivación. La magnitud de la puntuación de riesgo 
Tabla 4. Causas de hemorragia según grupo etario

\begin{tabular}{|c|c|c|c|c|c|}
\hline Edad & \multicolumn{5}{|c|}{ Orden de frecuencia } \\
\hline Adultos $>60$ años & $\begin{array}{l}\text { Enfermedad } \\
\text { diverticular }\end{array}$ & Angiodisplasia de colon & Colitis isquémica & Tumores de colon & $\begin{array}{l}\text { Pólipos y } \\
\text { poliposis }\end{array}$ \\
\hline Adultos $<60$ años & Ell & & $\begin{array}{l}\text { Pólipos y } \\
\text { poliposis }\end{array}$ & & \\
\hline $\begin{array}{l}\text { Adolescentes y } \\
\text { jóvenes }\end{array}$ & $\begin{array}{l}\text { Divertículo de } \\
\text { Meckel }\end{array}$ & Ell & $\begin{array}{l}\text { Pólipos y } \\
\text { poliposis }\end{array}$ & & \\
\hline
\end{tabular}

Ell: Enfermedad inflamatoria intestinal., Adaptada de Saperas, et al., $2007^{9}$

correlacionó con los desenlaces clínicos primarios, incluyendo necesidad de cirugía, requerimientos transfusionales, estancia hospitalaria y mortalidad.

La definición e identificación de factores y escalas de predicción clínica fiables permite tomar decisiones objetivas y sistematizadas, identificar correctamente a aquellos que requieren intervención endoscópica temprana y predecir resultados clínicos relevantes como la recurrencia de hemorragia o mortalidad. No existe evidencia suficientemente sólida para recomendar una escala de predicción clínica en particular, pero los siete factores clínicos descritos previamente han sido consistentes en la mayoría de los estudios.

\section{Tratamiento inicial de la hemorragia aguda de tubo digestivo bajo: restitución de volumen, transfusión y corrección de la coagulopatía}

El PRIMER PASO en el MANEJO de LA hemorRagia AGUDA DE TUBO DIGESTIVO BAJO ES EVALUAR EL ESTADO HEMODINÁMICO Y LA REANIMACIÓN INTENSIVA ANTES DE REALIZAR EL PROCEDIMIENTO ENDOSCÓPICO

Recomendación fuerte, calidad de evidencia moderada (GRADE 1B)

Debido a que los pacientes con HATDB suelen ser de edad avanzada y presentar múltiples comorbilidades, cuando se presentan con inestabilidad hemodinámica requieren tratamiento intrahospitalario y resucitación intravenosa intensiva con cristaloides (solución Hartmann preferentemente).

La estabilización hemodinámica del paciente corrige la hipovolemia, favorece una perfusión adecuada de los tejidos y previene la falla de uno o más órganos ${ }^{5,6,8}$.

En el 2004 Baradarian, et al. compararon los resultados de la resucitación intensiva y aquellos obtenidos con resucitación convencional en pacientes con hemorragia digestiva. El grupo que recibió resucitación intensiva presentó con menor frecuencia: infarto al miocardio $(p<0.05)$, tuvo una menor estancia en la $\mathrm{UCI}(p<0.05)$ y menor necesidad de intervención quirúrgica $(p<0.1)$. Así, la resucitación intensiva con cristaloides debe ser la conducta inicial en el tratamiento del paciente con hemorragia digestiva, pues disminuye de forma significativa la mortalidad ${ }^{21}$.

Semler, et al., en un estudio retrospectivo en pacientes ingresados a la $\mathrm{UCl}$ que recibieron cristaloides balanceados (solución Hartmann, lactato Ringer), observaron menor mortalidad hospitalaria (19.6 vs. $22.8 \%$; $p<0.01)$ comparándolos con aquellos a quienes se administró solución salina, la cual se asoció a una mayor frecuencia de lesión renal ${ }^{22}$.

La evidencia comentada sugiere que la resucitación intensiva (cristaloides) debe favorecerse sobre la resucitación estándar y debe ser la maniobra inicial (incluso antes de realizar colonoscopia) en el tratamiento de la HATDB, pues disminuye la mortalidad, infarto de miocardio y tiempo en la $\mathrm{UCl}^{5,6,8,21,22}$.

EN LA HEMORRAGIA AGUDA DE TUBO DIGESTIVO BAJO SE RECOMIENDA LA ESTRATEGIA DE TRANSFUSIÓN RESTRICTIVA

Recomendación fuerte, calidad de evidencia moderada (GRADE 1B)

Similar a las recomendaciones de la AMEG y otras sociedades internacionales, tanto en la HTDA como en la HATDB se recomienda una conducta transfusional restrictiva.

La transfusión sanguínea está indicada en pacientes con HATDB con inestabilidad hemodinámica. En aquellos sin comorbilidades el nivel meta de $\mathrm{Hb}$ es de $7 \mathrm{~g} / \mathrm{dl}$, mientras que cuando se presentan comorbilidades especialmente cardiovasculares el nivel meta de $\mathrm{Hb}$ es $9 \mathrm{~g} / \mathrm{dl}$. 
Estas recomendaciones representan una extrapolación de estudios en pacientes con HTDA, pues en estos no se incluyen pacientes con HATDB. La terapia transfusional restrictiva ha demostrado reducir significativamente la mortalidad y recurrencia de la hemorragia.

Los pacientes con evidencia clínica de hemorragia severa, que requieren transfusión de más de dos concentrados globulares y con comorbilidades importantes, deben de ser ingresados en $\mathrm{UCl}$ para monitoreo y vigilancia ${ }^{5,6,8,23-27}$.

Un metaanálisis de cinco estudios aleatorizados controlados que compararon la estrategia restrictiva (nivel meta $\mathrm{Hb} 7 \mathrm{~g} / \mathrm{dl}$ ) y la estrategia libre observaron menor riesgo de mortalidad (RR: 0.65; IC 95\%: 0.44-0.97; $p=0.03$ ) y menor recurrencia de la hemorragia (RR: 0.58; IC 95\%: 0.40-0.84; $p=0.004)^{9}$. Resultados similares fueron observados en un estudio que comparó la estrategia restrictiva (461 pacientes) y la liberal (460 pacientes), observando una supervivencia a seis semanas mayor en la estrategia restrictiva (95 vs. $91 \%$; HR: 0.55; IC 95\%: 0.33-092; $p=0.02$ ), así como mayores efectos adversos en la terapia liberal (48 vs. $40 \% ; p=0.02)^{28}$.

En conclusión, en pacientes con HATDB la transfusión de hemoderivados está indicada para obtener y mantener un nivel de $\mathrm{Hb}$ de $7 \mathrm{~g} / \mathrm{dl}$ (excepto en pacientes con comorbilidad cardiovascular cuyo nivel ideal es $9 \mathrm{~g} / \mathrm{dl}$ ). La estrategia de transfusión restrictiva es más costo-efectiva en la mayoría de los pacientes con HATDB.

\section{LA COAGULOPATÍA MODERADA (INR < 2.5) NO CONTRAINDICA LA ENDOSCOPIA DIAGNÓSTICA NI TERAPÉUTICA}

\section{Recomendación fuerte, calidad de evidencia mo- derada (GRADE 1B)}

Los antagonistas de la vitamina $\mathrm{K}$ (AVK), como la warfarina o la acenocumarina, y los anticoagulantes orales directos (ACOD), como el dabigatrán, el rivaroxabán y el apixabán, son frecuentemente utilizados como terapia antitrombótica y se asocian a un riesgo incrementado de hemorragia. Se estima que entre el 15 y el $32 \%$ de los pacientes que presentan un evento de HTD alto o bajo utilizan alguno de estos fármacos.

Aproximadamente el 15 y el $32 \%$ de los pacientes con hemorragia digestiva alta y baja respectivamente toman anticoagulantes ${ }^{30}$. La hemorragia gastrointestinal grave es una complicación seria de estos fármacos y ocurre aproximadamente en un $1-4 \%$ de las personas que los reciben, con una tasa de mortalidad del $10 \%$.

Comparados con la población general, los pacientes en tratamiento con warfarina tienen una incidencia de hemorragia severa tres veces mayor que la población en general y el tracto gastrointestinal es el sitio más común de hemorragia ${ }^{31}$.

Con respecto a los ACOD, el riesgo de hemorragia gastrointestinal es incierto y controvertido; existe evidencia de un metaanálisis que reportó un riesgo incrementado de hemorragia (OR: 1.45; IC 95\%: 1.07-1.97) en los usuarios de dabigatrán y rivaroxabán en comparación con los que usan warfarina ${ }^{32}$. Sin embargo, otro estudio indica que los pacientes en tratamiento con warfarina presentan un mayor riesgo de hemorragia gastrointestinal con respecto a pacientes con ACOD (12 vs. $9.9 \% ; p=0.002)^{33}$.

El manejo periendoscópico de la hemorragia gastrointestinal aguda en pacientes con anticoagulación plantea dificultades relacionadas tanto con el riesgo de hemorragia asociada a su uso como con el riesgo trombótico asociado a su interrupción, por lo que este grupo de pacientes deberá manejarse en conjunto con los especialistas encargados de la terapia antitrombótica.

\section{Revertir la anticoagulación antes del manejo endoscópico}

En pacientes con HTD aguda, clínicamente significativa (p. ej., hematoquecia severa que condiciona anemia aguda) en tratamiento con AVK se recomienda suspender estos últimos y corregir la coagulopatía.

Diversos estudios en pacientes con HTD han reportado que la coagulopatía (INR > 1.5 o TP prolongado $>3 \mathrm{~s}$ ) es el predictor clínico más importante de falla a la hemostasia endoscópica. ${ }^{30,31}$ La administraron de plasma fresco congelado (PFC) a sujetos con hemorragia gastrointestinal e ingesta de warfarina, con la meta de lograr un valor de INR entre 1.5 y 2.5 antes de la endoscopia urgente demostró una tasa de recurrencia y mortalidad similar a la observada en pacientes no anticoagulados ${ }^{34}$. Aunque el INR óptimo para que la terapia endoscópica sea segura y efectiva no se ha determinado, este estudio ha sido utilizado para proponer que con INR > 2.5 la endoscopia deberá preferentemente posponerse hasta lograr la reversión parcial o total de la coagulopatía, no así cuando el valor de INR es $\leq 2.5$. Las guías del Colegio Americano de Gastroenterología (ACG) consideran que un INR 
entre 1.5 y 2.5 es seguro y necesario para realizar hemostasia endoscópica efectiva ${ }^{5,31}$.

La reversión de la anticoagulación puede realizarse con la administración de vitamina $\mathrm{K}, \mathrm{PFC}$, concentrado de complejo de protrombina (CCP $3 F$ y $4 \mathrm{~F}$ ) y factor VIlla recombinante activado (rFVIla) $)^{30,31,35}$.

Para los agentes AVK, la administración de 5-10 mg iv. de vitamina $\mathrm{K}$ logra la disminución del INR a partir de las 2-4 $\mathrm{h}$ y este se normaliza dentro de las siguientes $24 \mathrm{~h}$. La vitamina $\mathrm{K}$ no es ideal para la reversión urgente, pero proporciona una corrección sostenida de la coagulopatía. Se recomienda determinación del valor de INR cada $12 \mathrm{~h}$ hasta lograr la corrección. De ser necesario se puede repetir la administración de la vitamina $\mathrm{K}^{32,35}$.

El PFC se recomienda a dosis de $15-30 \mathrm{ml} / \mathrm{kg}$. Se requieren aproximadamente 3-5 unidades en situaciones de reversión urgente; el efecto parcial se comienza a observar después de algunas horas tras su administración y se necesitan al menos nueve horas para lograr una reversión completa $(\text { INR }<1.5)^{31,35}$.

En todos los pacientes excepto aquellos con sangrado rectal menor o leve (hematoquecia escasa autolimitada que no causa anemia) se deben suspender los AVK y administrarse vitamina $K$, pero se requiere de una reversión urgente, por lo que se puede administrar vitamina $\mathrm{K}$ v.o. (1-5 mg) o por vía iv. a dosis bajas (1-2.5 mg), especialmente con valores de INR $\geq 5^{31}$.

En pacientes con hemorragia severa e inestabilidad hemodinámica persistente 0 intermitente se deben administrar factores de coagulación, incluso en el caso de INR en rangos terapéuticos; se recomienda utilizar en promedio 4F-CCP en lugar de PFC. EI INR debe medirse 20-30 minutos después y si permanece $\geq 1.5$ se debe administrar otra dosis del CCP. Tras esta segunda dosis, el INR se debe medir nuevamente 6-8 $\mathrm{h}$ después y posteriormente una vez al día mientras la situación continúe siendo crítica ${ }^{31,35}$.

La coadministración iv. de vitamina $\mathrm{K}$ es necesaria para prevenir una "coagulopatía de rebote», que puede ocurre 12-24 h después de la normalización del INR una vez que se han eliminado los factores transfundidos, ya que la vida media de la warfarina es de 20 $60 \mathrm{~h}$, la del CCP de 6-8 h y la del PFC de $1.5 \mathrm{~h}-2 \mathrm{~d}^{31}$.

Las pruebas de coagulación ordinarias no son fiables o útiles para medir el efecto anticoagulante de los ACOD. En ausencia de insuficiencia hepática o renal Ios ACOD pierden gradualmente su efecto anticoagulante entre 12 y $24 \mathrm{~h}$ después de la última dosis ${ }^{30,31,35}$. Respecto al tratamiento de la hemorragia gastrointestinal en personas que utilizan ACOD se ha propuesto que en hemorragia clínicamente significativa se debe hidratar adecuadamente al paciente, pues esto mejora la excreción renal; además se debe iniciar terapia transfusional cuando sea necesario, con la finalidad de mantener la estabilidad hemodinámica, pues considerando lo corto que es su vida media, el tiempo es el principal antídoto contra el efecto de los $\mathrm{ACOD}^{30,31,32,35}$. No debe utilizarse vitamina $\mathrm{K}$ ni PFC como antídoto para estos.

Si el paciente está hemodinámicamente estable y responde a las medidas de resucitación, es recomendable vigilar y diferir la endoscopia de 12 a $24 \mathrm{~h}$, dando oportunidad a la depuración del fármaco y consecuente restablecimiento de la coagulación. En hemorragia aguda con inestabilidad hemodinámica persistente y potencialmente mortal en que la endoscopia de urgencia es necesaria, el caso debe ser manejado por un equipo multidisciplinario y considerar el uso de agentes prohemostásicos (4F-CCF o rFVIIa) 31,32,33,35.

En conclusión, el tratamiento del paciente con HTDB y terapia antitrombótica requiere individualizarse y valoración por un equipo multidisciplinario (Tabla 5).

\section{Hemorragia de tubo digestivo bajo de origen en colon}

\section{Papel de la colonoscopia en la hemorragia aguda de tubo digestivo bajo}

\section{LA COLONOSCOPIA ES EL MÉTOdO DIAGNÓSTICO DE ELECCIÓN EN PACIENTES CON HEMORRAGIA AGUDA DE TUBO DIGESTIVO BAJO CON SOSPECHA DE ORIGEN EN COLON}

\section{Recomendación fuerte, calidad de evidencia mo- derada (GRADE 1B)}

Aunque la mayoría de los casos de HATDB ceden sin intervención, la colonoscopia ha demostrado su utilidad como procedimiento diagnóstico de elección en la mayoría de los pacientes con HATDB, intermitente o crónica ${ }^{6,36}$. En la HATDB que se presenta con hematoquecia, la colonoscopia se recomienda en la evaluación inicial de pacientes que se encuentran hemodinámicamente estables y que, por lo tanto, pueden ser sometidos a la preparación intestinal requerida. Si hay sospecha de hemorragia proximal se puede realizar primero una endoscopia de tubo digestivo alto y si esta es negativa se realizará la colonoscopia previa preparación $6,37,38$.

La colonoscopia en la HATDB tiene múltiples ventajas comparada con otros procedimientos, como un 
Tabla 5. Reversión de la anticoagulación en la hemorragia aguda de tubo digestivo bajo previo a la endoscopia

\begin{tabular}{|c|c|}
\hline Revertir con* & ¿Cuándo?/¿En qué situación?† \\
\hline Vitamina $\mathrm{K}$ & Hemorragia grave o INR $>2.5$ por AVK y en cirróticos \\
\hline PFC & Hemorragia grave por AVK \\
\hline PCC & Solo cuando se requiera rápida reversión, en hemorragia grave. Alto riesgo tromboembólico \\
\hline Factor VIla & Limitado a hemorragia grave potencialmente mortal, para la reversión de ACOD. Alto riesgo tromboembólico \\
\hline
\end{tabular}

excelente perfil de seguridad (tasa de complicaciones del $0.3-2 \%$ ) y alto rendimiento diagnóstico (74-100\%) y terapéutico (10-40\% de los pacientes, logrando hemostasia inmediata en el 50-100\% de los casos). El rendimiento diagnóstico y terapéutico es superior, pues no requiere hemorragia activa para establecer un diagnóstico etiológico ${ }^{5,8,37-39}$.

Las desventajas de la colonoscopia en el manejo de la HTDB activa incluyen su naturaleza invasiva, la necesidad de preparación y de personal especializado (endoscopista, anestesiólogo, asistente en endoscopia), además de que los estigmas de hemorragia no son fácilmente detectables. Las complicaciones asociadas son poco frecuentes, pero potencialmente graves. En una revisión de cuatro estudios con 664 pacientes, reportaron dos perforaciones, con una tasa general de complicaciones del $0.3 \%$ para la colonoscopia y del $0.6 \%$ para la colonoscopia realizada con urgencia. Otros riesgos potenciales incluyen insuficiencia cardíaca congestiva secundaria a sobrecarga de volumen, anomalías electrolíticas y neumonía por aspiración ${ }^{37,39}$.

Durante la colonoscopia en la HTDB es importante llevar a cabo una inspección detallada de la mucosa tanto durante la inserción como a la retirada del colonoscopio; se recomienda preparación intestinal adecuada, así como aspiración de restos hemáticos y fecales. El empleo de bomba de irrigación facilita la limpieza del residuo adherido ${ }^{5}$. Algunas publicaciones que evaluaron la seguridad de la colonoscopia en el postoperatorio inmediato han demostrado que la técnica de inmersión con agua puede favorecer la identificación de lesiones sangrantes, puesto que hace evidente el punto de origen de la hemorragia ${ }^{40}$. La colonoscopia continúa siendo el estándar de oro para el abordaje inicial, tanto diagnóstico como terapéutico, en la HTDB.

\section{Colonoscopia temprana en hemorragia aguda de tubo digestivo bajo}

\section{EN PACIENTES HEMODINÁMICAMENTE ESTABLES CON HEMORRAGIA AGUDA DE TUBO DIGESTIVO BAJO PERSISTENTE SE RECOMIENDA LA COLONOSCOPIA TEMPRANA (EN LAS PRIMERAS 24 H), POSTERIOR A UNA PREPARACIÓN DEL COLON}

\section{Recomendación débil, calidad de evidencia mo- derada (GRADE 2B)}

La colonoscopia temprana es aquella que se realiza dentro de las primeras $24 \mathrm{~h}$ de la admisión del paciente. Aunque algunos metaanálisis y guías clínicas limitan esta recomendación a pacientes con datos clínicos de alto riesgo o evidencia de hemorragia persistente, existe información que sugiere que la colonoscopia temprana puede ser una medida apropiada incluso en los pacientes sin datos sugestivos de hemorragia severa o persistente ${ }^{5,38,39}$. Múltiples estudios demuestran que la colonoscopia temprana comparada con la colonoscopia electiva y otros procedimientos es segura y efectiva ${ }^{7,41-43}$, además de incrementar la identificación (RM: 4.12; IC 95\%: 2.0-8.49) $7,41,43-48$ y tratamiento efectivo de las lesiones (RM: 4.17; IC 95\%: 3.2-7.49) $44,45,48,49$ causantes de la hemorragia, reduce el tiempo $41-44,46,48,50$ y los costos de hospitalización (22,142 vs. 28,749 dólares americanos) ${ }^{50}$.

Sin embargo, hacerla de manera temprana no ha demostrado reducir la mortalidad (RM: 0.61; IC 95\%: $0.12-3.23)^{41,42,44,47-51}$, disminuir la hemorragia recurrente (RM: 0.89; IC 95\%: 0.49-1.62) $42,44,45,47,48,51$, los requerimientos transfusionales (RM: -0.27 ; IC 95\%: -1.60 a $1.06)^{42,44,45,47,48,51}$ ni la necesidad de cirugía (RM: 0.78 ; IC 95\%: 0.39-1.56) ${ }^{48}$. La revisión sistemática y metaanálisis reciente de Afshar, et al. ${ }^{48}$ demostró que la colonoscopia temprana sí tiene un impacto positivo, ya que establece el diagnóstico definitivo de la lesión 
sangrante con más frecuencia (RM: 4.12; IC 95\%: 2.08.49) y se asocia con una menor estancia hospitalaria (-1.52 días; IC 95\%: -2.54 a -0.50 días) al ser comparada con colonoscopia electiva $u$ otras estrategias diagnósticas y terapéuticas ${ }^{48}$.

Una limitante para la interpretación de resultados es que en su mayoría se trata de estudios retrospectivos, observacionales, comparativos y estudios clínicos aleatorizados con un número reducido de casos en los que incluso hay heterogeneidad en la definición de HTDB, así como en la definición de colonoscopia urgente, temprana y electiva.

En el caso de HATDB masiva con deterioro hemodinámico importante, cuando no es posible estabilizar de manera temprana al paciente se recomienda la angiografía por tomografía o angiografía mesentérica con embolización. Por otra parte, en el caso de pacientes con HATDB en quienes haya remitido la hemorragia o pacientes de bajo riesgo que se encuentren hemodinámicamente estables, se puede considerar colonoscopia electiva (posterior a las $24 \mathrm{~h}$ ) cuando por algún motivo logístico sea más conveniente ${ }^{5,43}$.

Al analizar la evidencia disponible, y tomando en cuenta que el rendimiento de la colonoscopia aumenta al realizarla durante hemorragia activa en comparación con colonoscopia electiva (de 20 a $85 \%$ (39 $^{39}$, el grupo considera que la evidencia apoya el realizar colonoscopia temprana en la mayoría de los pacientes con HATDB persistente sin deterioro hemodinámico, a excepción de los casos en los que no hubiera disponibilidad de recursos humanos o materiales apropiados.

\section{Características de la preparación de colon en hemorragia aguda de tubo digestivo bajo}

\section{LA PREPARACIÓN ENÉRGICA DEL COLON INCREMENTA EL RENDIMIENTO DIAGNÓSTICO Y TERAPÉUTICO EN HEMORRAGIA AGUDA DE TUBO DIGESTIVO BAJO}

Recomendación fuerte, calidad de evidencia moderada (GRADE 1B)

La preparación intestinal es indispensable para la adecuada visualización de la mucosa colónica ${ }^{38}$. Las colonoscopias sin preparación han demostrado bajas tasas de canulación del ciego (60\%) e incremento en el riesgo de perforación por mala visualización, por lo que no se recomiendan ${ }^{52,53}$.

Una preparación enérgica del colon consiste en la administración oral o por sonda nasogástrica de hasta 6 I de polietilenglicol en un periodo de 3-4 $\mathrm{h}$ hasta que las evacuaciones sean claras y sin restos hemáticos y/o fecales. Se debe considerar que esta preparación puede aumenta el riesgo de vómito, neumonía por aspiración y sobrecarga de volumen ${ }^{37,54}$, pero permite el diagnóstico hasta en un $74 \%$ de los pacientes y el tratamiento endoscópico un $39 \%{ }^{55}$.

En un estudio prospectivo aleatorizado de Green, et al. se demostró que con la preparación de alto volumen (4-6 I de polietilenglicol) v.o. o vía sonda nasogástrica en un periodo de 3-4 h se encontró el diagnóstico definitivo y presuntivo en el 42 y el $54 \%$ de los casos respectivamente ${ }^{47}$.

Algunos pacientes no son capaces de tolerar una preparación rápida de alto volumen v.o., por lo que se puede utilizar preparación por sonda nasogástrica. En el estudio publicado por Jensen, et al. de 121 pacientes con sangrado diverticular, el $33 \%$ de los pacientes recibieron 5-6 I de preparación a base de sulfatos por sonda nasogástrica para limpieza del colon en un periodo de 3-4 $\mathrm{h}^{56}$.

Durante la reunión el grupo consideró que ingerir preparación de alto volumen es poco tolerado por el paciente, por lo que se puede considerar preparación de menor volumen siempre y cuando se obtengan evacuaciones claras sin restos hemáticos o fecales.

\section{Características del tratamiento endoscópico de hemorragia aguda de tubo digestivo bajo originada en el colon}

La SELECCIÓN DEL TRATAMIENTO DE LA HEMORRAGIA AGUDA DE TUBO DIGESTIVO BAJO DEPENDERÁ DE LOS HALLAZGOS ENDOSCÓPICOS, LOS CUALES PUEDEN CLASIFICARSE EN DE ALTO Y BAJO RIESGO

\section{Recomendación fuerte, calidad de evidencia baja (GRADE 1C)}

Las lesiones se deben de clasificar en de alto y bajo riesgo, ya que esto impacta en la selección del tratamiento. Las lesiones de alto riesgo son aquellas con estigmas de hemorragia reciente (EHR) y se definen por la presencia de hemorragia activa, coágulo adherido o vaso visible ${ }^{41,56}$. Las lesiones de bajo riesgo son aquellas que no tienen dichos estigmas o que se originan en procesos inflamatorios (Tabla 6). La selección del tratamiento se discutirá en segmentos posteriores ${ }^{41,56}$. Las lesiones que se asocian con mayor riesgo de hemorragia del TDB son los divertículos, las angioectasias y el sangrado pospolipectomía ${ }^{57}$. 
Tabla 6. Clasificacion de lesiones

\begin{tabular}{|l|l|}
\hline Alto grado & Bajo grado \\
\hline Hemorragia activa & Lesiones no vasculares \\
\hline Coágulo adherido & Hemorragia por Ell \\
\hline Vaso visible & Pólipos/tumores \\
\hline
\end{tabular}

Ell: enfermedad inflamatoria intestinal.

Debe llevarse a cabo tratamiento endoscópico HEMOSTÁTICO EN LESIONES CON ESTIGMAS DE ALTO RIESGO O HEMORRAGIA ACTIVA

\section{Recomendación fuerte, calidad de evidencia baja (GRADE 1C)}

Una vez que se detectan lesiones con estigmas de alto riesgo, se debe ofrecer tratamiento endoscópico. La elección del método hemostático dependerá de la causa y localización del sangrado, la dificultad para llegar al sitio de sangrado y la experiencia del endoscopista ${ }^{8}$.

No existe evidencia de alta calidad sobre la eficacia y seguridad de la hemostasia endoscópica en la HTDB. Los estudios disponibles incluyen estudios observacionales y series de casos, en su mayoría en hemorragia diverticular.

Se ha reportado una tasa de éxito de hemostasia endoscópica del $92 \%$, una tasa de recurrencia temprana del $8 \%$ y de recurrencia tardía del $12 \%$. Esto se ha tratado de explicar con el hecho de que en la HTDB no existe terapia médica (p. ej., inhibidores de la bomba de protones), al contrario de en la HTDA, en la cual disminuye las tasas de hemorragia recurrente ${ }^{56}$.

El éxito de la colonoscopia en hemostasia pospolipectomía es del $22 \%$, con un número necesario a tratar de 4.5 pacientes $^{58}$. La efectividad en otras etiologías se extrapola en casos de HTDA ${ }^{59}$.

Durante la elaboración de la guía, se consensuó que al encontrar alguna lesión de alto riesgo se debe realizar terapia endoscópica con la finalidad de evitar recurrencia de la hemorragia.

\section{EL EMPLEO DE INYECCIÓN DE ADRENALINA PUEDE SER} ÚTIL PARA LOGRAR HEMOSTASIA INICIAL O PARA MEJORAR LA VISUALIZACIÓN DE LAS LESIONES SANGRANTES

\section{Recomendación fuerte, calidad de evidencia alta (GRADE 1A)}

El empleo de inyección de adrenalina puede ser útil para lograr hemostasia inicial y/o para mejorar la visualización de lesiones sangrantes ${ }^{60}$. La inyección de adrenalina 1:10,000 provoca vasoconstricción local y taponamiento físico del vaso; esto facilita la visualización en caso de hemorragia activa, pues se puede localizar con mayor facilidad el sitio a tratar con otro método y así lograr hemostasia inicial. Se debe evitar la sobreinyección para no comprometer el sitio a $\operatorname{tratar}^{38}$.

\section{EN HEMORRAGIA POSPOLIPECTOMÍA LAS TERAPIAS DE INYECCIÓN, TÉRMICAS Y MECÁNICAS SON OPCIONES TERAPÉUTICAS ÚTILES}

\section{Recomendación fuerte, calidad de evidencia alta (GRADE 1A)}

La hemorragia es la complicación más frecuente posterior a la polipectomía, con una frecuencia que varía entre el 0.1 y el $0.6 \%{ }^{61}$. Esta puede ser temprana (primeras 24 h) 0 tardía (1 a 14 días) ${ }^{62}$. El riesgo se incrementa hasta en un $11 \%$ cuando se realiza resección endoscópica de la mucosa (Tabla 7) ${ }^{63-70}$.

Para evitar hemorragia pospolipectomía tanto temprana como tardía se debe identificar el o los factores de riesgo y de acuerdo a ellos se seleccionará el o los métodos profilácticos, como inyección del tallo grueso o de la base del pólipo pediculado, así como la aplicación de hemoclip en el sitio de la polipectomía.

En pólipos sésiles de $10 \mathrm{~mm}$ o menores es seguro realizar la polipectomía con asa fría. En aquellos entre 10-19 mm se sugiere la polipectomía con asa caliente después de la inyección submucosa. En lesiones sésiles que miden entre 10-19 mm y donde existe el riesgo de efectos adversos (localización en el ciego o colon ascendente, riesgo alto de hemorragia) es útil la polipectomía con asa fría con o sin inyección submucosa previa, y hemostasia mecánica antes de polipectomía para pólipos pediculados grandes con tallo mayor a $10 \mathrm{~mm}$ o cabeza mayor a $20 \mathrm{~mm}^{70-75}$.

El tratamiento de la hemorragia pospolipectomía depende del tiempo de presentación. En la hemorragia 
Tabla 7. Factores de alto riesgo para hemorragia pospolipectomía ${ }^{4-11}$

\begin{tabular}{|c|c|}
\hline Pólipo & Paciente \\
\hline 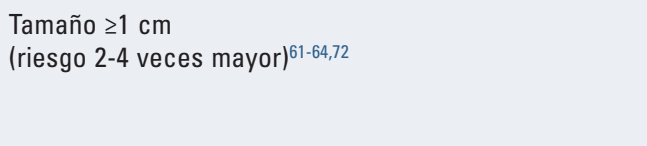 & $\begin{array}{l}\text { Edad } \geq 65 \text { años } \\
\text { Hipertensión arterial } \\
\text { Enfermedad cardiovascular } \\
\text { Enfermedad renal| }{ }^{61,62,63}\end{array}$ \\
\hline $\begin{array}{l}\text { Localización: colon derecho } \\
\text { (riesgo } 2.6 \text { a } 4.6 \text { mayor) }{ }^{61,64-66}\end{array}$ & $\begin{array}{l}\text { Anticoagulantes } \\
\text { Previos (riesgo } 3.7 \text { mayor) }{ }^{61} \\
\text { Ininterrumpido ( } 5.6 \text { mayor) }^{70}\end{array}$ \\
\hline $\begin{array}{l}\text { Morfología e histología } \\
\text { Pediculado con tallo grueso } \\
\text { Lesión lateralmente extendida } \\
\text { Adenoma, componente velloso y adenocarcinoma }{ }^{61,67-69} \\
\text { Paris } 0 \text {-IIa + Is en REM }{ }^{65}\end{array}$ & Cirrosis descompensada (Child Pugh B, C) (riesgo 3.5 mayor) ${ }^{64}$ \\
\hline
\end{tabular}

REM: Resección endoscópica de la mucosa

temprana la presión sostenida del asa de polipectomía en la base del pedículo habitualmente logra hemostasia; si esto no es posible se recomienda el uso de inyección con adrenalina $(1: 10,000)$ en la base del pólipo para detener la hemorragia o para visualizar mejor y adicionar un método mecánico como hemoclip, endoloop o liga ${ }^{61}$. La monoterapia con adrenalina ha demostrado ser útil en control de la hemorragia (RM: 0.37), mientras que la terapia combinada de adrenalina con método hemostático mecánico o térmico tiene una efectividad de mayor (RM: 0.12); sin embargo, la mayoría de los estudios han sido publicados en HTDA ${ }^{61}$.

Cuando se utiliza hemoclip, este debe ser aplicado en el punto de hemorragia. Se puede también usar un asa cuando la longitud del tallo residual lo permita ${ }^{61,76,77}$. Si se usa un método térmico se sugiere la sonda caliente (Heater probe ${ }^{\circ}$ ) con $15 \mathrm{~J}$ durante 3-5 s o sonda de coagulación bipolar con 12 a $15 \mathrm{~W}^{77,78}$.

Si se utiliza la punta del asa de polipectomía se debe tener cuidado para evitar daño térmico por corriente monopolar. El uso de pinza de coagulación monopolar se recomienda en disección endoscópica de la submucosa para hemostasia de vasos submucosos y en algunos casos de resección endoscópica de la mucosa. La coagulación de no contacto con plasma argón (APC) puede extender la profundidad de daño térmico, por lo que debe utilizarse con precaución ${ }^{78}$.

La hemorragia tardía suele originarse en las úlceras desarrolladas en el sitio de la polipectomía. Cuando estas presentan base limpia, con fibrina o mancha hemática, no se requiere tratamiento endoscópico.

Ante la presencia de vaso visible, coágulo adherido y hemorragia activa, se recomienda terapia endoscópica, preferentemente combinando adrenalina y un método mecánico o térmico ${ }^{61}$. En caso de utilizar hemoclip, este debe ser colocado directamente en el vaso sangrante 0 en la base completa de la polipectomía. Cuando la base se encuentre indurada, se pueden considerar métodos mecánicos como clip OTSC (Ovesco $\left.{ }^{\circledR}\right)^{79,80}$ o endosutura (over Stitch Apo$\left.1 / 0^{\circledR}\right)$, térmicos o polvos hemostáticos ${ }^{81,82}$. Si la terapia endoscópica falla debe considerarse la embolización mediante arteriografía o tratamiento quirúrgico $0^{61}$.

La profilaxis de hemorragia pospolipectomía mediante la colocación de un asa hemostásica e inyección de adrenalina en la base del tallo de los pólipos pediculados mayores a $2 \mathrm{~cm}$ ha demostrado disminuir la incidencia de hemorragia temprana en pacientes con pólipos grandes cuando se compara con monoterapia con adrenalina ( 2.3 vs. $10.6 \% ; p=0.04)^{73,75}$, aunque un metaanálisis reciente encontró que la monoterapia con inyección o la monoterapia mecánica reducen el riesgo de hemorragia temprana (RR: 0.32, IC 95\%: 0.11-0.67 para inyección y RR: 0.13, IC 95\%: 0.03-0.37 para método mecánico, respectivamente) y la terapia combinada no fue superior a estas. Ninguna terapia profiláctica disminuyó el riesgo de hemorragia tardía ${ }^{83}$.

\section{LA COLITIS ISQUÉMICA, LA COLITIS INFECCIOSA, LA NEOPLASIA COLORRECTAL Y LA ENFERMEDAD \\ INFLAMATORIA INTESTINAL SE ASOCIAN CON HEMORRAGIA DIFUSA;' SIN EMBARGO, LA PERTINENCIA DEL MANEJO ENDOSCÓPICO DEBE INDIVIDUALIZARSE}

\section{Recomendación fuerte, calidad de evidencia baja (GRADE 1C)}

La colitis isquémica suele afectar principalmente a personas de la tercera edad con ateroesclerosis 
importante y/o cardiopatía, así como otras condiciones que pueden disminuir temporalmente el riego sanguíneo mesentérico debido a vasoespasmo o disminución en la presión sanguínea. Una de sus manifestaciones clínicas es la hemorragia; aunque los episodios de sangrado suelen autolimitarse en la mayoría de los casos, estos se asocian con un elevado riesgo de mortalidad $^{6,84}$. Las zonas más afectadas son aquellas con bajo flujo (ángulo esplénico y unión rectosigmoidea).

Los pacientes con enfermedad inflamatoria intestinal (EII) pueden presentar sangrados graves, con una incidencia reportada de 0.1 a $1.2 \%{ }^{85}$; similar a los casos con isquemia, la hemorragia se autolimita en cerca de la mitad de los casos, pero hasta un tercio de estos puede recurrir. Otras alteraciones de la mucosa como infecciones (virus de inmunodeficiencia humana [VIH], citomegalovirus, bacterias) y neoplasias (linfoma, sarcoma de Kaposi) también suelen presentarse con sangrados que habitualmente desaparecen espontáneamente, aunque la tasa de mortalidad asociada es cercana al $15 \%$, esencialmente en pacientes con $\mathrm{VIH}^{85-87}$.

Las neoplasias colorrectales son la causa de cerca del $10 \%$ de los episodios de hematoquecia, anemia por deficiencia de hierro y/o de HTDB crónica. Pueden presentar sangrados intermitentes, que se autolimitan, o bien prolongarse si se asocian con el uso de antiagregantes plaquetarios y/o anticoagulantes. Solo del 6.5 al $20 \%$ de las hemorragias asociados a tumores 0 lesiones de la mucosa requieren tratamiento endoscópico ${ }^{57}$. Para lograr hemostasia en lesiones asociadas a neoplasia se recomiendan métodos como electrocoagulación bipolar y sonda caliente.

Aunque con evidencia de baja calidad, se puede considerar el uso de láser, agentes esclerosantes y polvos hemostásicos. En las lesiones originadas por alteraciones en la mucosa se prefiere la inyección de adrenalina, clips y polvos hemostásicos, con una elevada efectividad y baja tasa de complicaciones asociadas $^{88-92}$. El uso de fármacos como el ácido tranexámico no ha demostrado efectividad en la hemostasia de HTDB originada por estas etiologías ${ }^{93}$. Aquellos casos refractarios o recurrentes deberán someterse a embolización angiográfica y/o cirugía.

Debido a que la información disponible en estas patologías proviene solo de series de casos, la elección de tratamiento deberá individualizarse, considerar la experiencia y recursos disponibles, así como la preferencia del endoscopista ${ }^{94}$. El papel de la colonoscopia en la HTDB asociada a colitis isquémica, neoplasia colorrectal y Ell es frecuentemente diagnóstico, pues a excepción de la hemorragia por neoplasia, el tratamiento definitivo es médico y/o quirúrgico.

\section{Hemorragia de tubo digestivo de origen anorrectal}

\section{Exploración física y estudios complementarios}

\section{LA EXPLORACIÓN ANORRECTAL Y LA ANOSCOPIA ESTÁN INDICADAS EN PRESENCIA DE RECTORRAGIA}

\section{Recomendación fuerte, calidad de evidencia mo- derada (GRADE 1B)}

La sospecha de rectorragia se establece ante la presencia de sangre roja rutilante durante la defecación, en forma de gotas o chorro sin coágulos, o con manchado en el papel durante el aseo. Su evaluación debe iniciar estableciendo sus características clínicas como tiempo de evolución (aguda o crónica), magnitud y repercusión hemodinámica, además realizar la exploración física de la región.

La presencia de dolor anal es altamente sugerente de etiología de localización distal ${ }^{95}$. Otros factores que sugieren origen anorrectal son la edad, antecedente de cáncer colorrectal, de próstata o cervicouterino, Ell, insuficiencia renal o hepática, ingesta crónica de medicamentos como AINE o anticoagulantes, patrón defecatorio, tipo de dieta, viajes recientes y prácticas sexuales $^{95,96}$.

La exploración física proctológica es un método diagnóstico que consta de tres etapas: la inspección anoperineal, el tacto rectal y la endoscopia anorrectal (anoscopia o rectosigmoidoscopia).

Se debe explicar al paciente el procedimiento y realizarlo en un área aislada, confortable y con iluminación adecuada. La inspección anoperineal incluye la visualización directa del ano, la región perianal, el periné y ambos glúteos, para descartar la presencia de hemorroides, fisura anal, úlceras, orificios fistulosos, dermatosis, cicatrices o alguna tumoración; se le solicita al paciente un esfuerzo de «pujo» para evidenciar algunas patologías como el síndrome del perineo descendente, la dilatación de hemorroides externas o el prolapso de hemorroides internas o alguna neoplasia rectal baja. El tacto rectal se realiza con un guante y el dedo índice bien lubricado, con movimientos suaves y gentiles, palpando la región perianal para descartar dolor, definir el tono de los esfínteres, la integridad de las paredes del conducto anal y la correcta contracción de los músculos. En ocasiones se 
requiere sedación o analgesia en presencia de dolor intenso pero nunca se debe obviar la exploración física anorrectal.

La anoscopia es un procedimiento de bajo costo, rápido, sencillo que no requiere de preparación intestinal. Se utiliza un anoscopio de $7 \mathrm{~cm}$ de longitud para la visualización directa del conducto anal y de la parte inferior del recto. Se debe hacer una inspección circular completa. Inmediatamente después del tacto rectal se introduce el anoscopio bien lubricado, retirándolo en forma suave para descartar neoplasias o patologías intraanales o la presencia de estigmas de hemorragia. Se pide al paciente que puje para observar el prolapso de las hemorroides o de la mucosa $a^{95,97,98}$.

LOS ESTUDIOS COMPLEMENTARIOS DE LA EXPLORACIÓN ANORRECTAL INCLUYEN LA RECTOSIGMOIDOSCOPIA FLEXIBLE, LA RECTOSIMOIGDOSCOPIA RÍGIDA Y LA COLONOSCOPIA

\section{Recomendación fuerte, calidad de evidencia mo- derada (GRADE 1B)}

La sigmoidoscopia flexible permite la visualización de los últimos $60 \mathrm{~cm}$ del colon y recto y es más confortable para los pacientes, aunque requiere preparación intestinal previa con laxantes y/o enemas, personal entrenado y con experiencia, y no requiere sedación de manera sistemática. Es un procedimiento seguro, sensible y específico. Su principal desventaja es que no explora por completo el colon, por lo que solo diagnostica aproximadamente el $50 \%$ de las neoplasias colorrectales ${ }^{99,100}$.

Si después de la anoscopia de urgencia por rectorragia se observa el ámpula rectal vacía se puede considerar una rectosigmoidoscopia rígida de manera inmediata para aumentar la sensibilidad diagnósti$\mathrm{ca}^{101,102}$. La rectosigmoidoscopia rígida de $25 \mathrm{~cm}$ permite la visualización de la parte más distal del sigmoides, la unión rectosigmoidea y el recto. Es un procedimiento rápido y seguro, de bajo costo y rara vez precisa sedación; por lo regular se requiere preparación intestinal con enemas evacuantes. La sigmoidoscopia rígida es menos tolerada que la flexible, por producir mayor incomodidad (29.7 vs. $10.1 \%$ ), ansiedad (27.6 vs. $9.8 \%$ ) y molestias de pudor a los pacientes (12.8 vs. $5.2 \%)^{103}$.

Un estudio de costo-efectividad sugiere que ante una rectorragia en pacientes jóvenes menores de 25 años, la anoscopia seguida de sigmoidoscopia debe preferirse a la colonoscopia, excepto en pacientes con anemia $u$ otros datos de alarma ${ }^{104}$.

\section{Etiologías de la hemorragia del tubo digestivo bajo de origen anorrectal}

\section{Para el tratamiento endoscópico de la} HEMORRAGIA DEL TUBO DIGESTIVO BAJO POR PROCTOPATÍA POR RADIACIÓN SE RECOMIENDA EL USO DE PLASMA ARGÓN

\section{Recomendación fuerte, calidad de evidencia mo- derada (GRADE 1B)}

La lesión colónica inducida por radiación ocurre en el $20 \%$ de los pacientes sometidos a radioterapia, siendo el recto y el colon distal las áreas más susceptibles debido a la inmovilidad relativa de estos segmentos y a las dosis de radiación empleadas para las neoplasias pélvicas. Sus manifestaciones clínicas incluyen el dolor anorrectal, la diarrea y el sangrado rectal evidente o manifestado por anemia. El sangrado anorrectal rara vez pone en peligro la vida y suele resolverse de manera espontánea. Los hallazgos endoscópicos incluyen pérdida de la vasculatura de la mucosa, eritema en parches y angiectasias que correlacionan con endarteritis isquémica de las arteriolas submucosas y fibrosis de la submucosa.

La mayoría de los pacientes desarrollan los datos clínicos después de uno a dos años de la exposición a la radiación, pero se pueden presentar hasta 30 años después. Cuando la proctopatía por radiación se encuentra de manera incidental en una endoscopia, no requiere tratamiento.

En pacientes con síntomas moderados y en la fase inflamatoria aguda se ha empleado terapia conservadora con la instilación rectal de sucralfato mostrando cicatrización y mejoría de los síntomas. Por otra parte, los enemas de ácido 5-aminosalicílico o de ácidos grasos de cadena corta no han probado eficacia, mientras que el oxígeno hiperbárico se ha asociado a mejoría significativa ${ }^{105}$.

En la proctopatía crónica, aquella que se presenta dos meses después de la radioterapia o la etapa aguda, la coagulación con APC es el estándar de la terapia endoscópica. El APC está casi universalmente disponible, es de relativo bajo costo y ofrece la posibilidad de aplicación tangencial y en retroflexión, lo que suma a su aplicación y efectividad clínica con una eficacia del $80-90 \%$ para lograr hemostasia.

Para su aplicación se recomienda una preparación completa del colon antes de emplear este método. Se sugiere utilizar 25-60 W de energía y flujos de 0.5-2.5 l/min. En la mayoría de los casos se requiere de una a tres sesiones. Su uso se ha asociado a 
complicaciones como estenosis rectal, fístula rectovaginal, tenesmo, dolor rectal, perforación y explosión durante el procedimiento ${ }^{106,107}$.

\section{Otras alternativas de tratamiento endoscópico}

DE LA HEMORRAGIA DEL TUBO DIGESTIVO BAJO

SECUNDARIA A PROCTOPATÍA POR RADIACIÓN INCLUYEN LA APLICACIÓN TÓPICA DE FORMALINA (2-10\%), LA ABLACIÓN POR RADIOFRECUENCIA, LA CRIOTERAPIA Y LA TERAPIA CON LÁSER

\section{Recomendación débil, calidad de evidencia baja (GRADE 2C)}

La terapia de ablación con radiofrecuencia parece que conduce a la resolución completa de las lesiones en los pacientes con proctopatía crónica por radiación, con mejoría de los índices clínicos y endoscópicos y sin efectos adversos mayores, pero se requieren más estudios para confirmarlo ${ }^{108,109}$.

La crioablación consiste en la aplicación de nitrógeno líquido o dióxido de carbono gaseoso en estado aerosolizado (espray) sobre la mucosa afectada. Sus ventajas incluyen que es un método de no contacto que puede ser aplicado bajo visión directa y dirigida con precisión a las áreas afectadas sin provocar daño significativo a tejidos más profundos. En un estudio prospectivo de 10 pacientes se mostró su efectividad para inducir mejoría endoscópica y sintomática en el 80 y $70 \%$ de los pacientes respectivamente ${ }^{110}$.

La aplicación de formalina diluida entre un 4 y un $10 \%$ mediante instilación o con aplicación a través de hisopos ha reportado tasas de efectividad de hasta el $80 \%$ en el control de sangrado rectal, y una tasa de complicaciones del $4 \%$ (necrosis rectal, fístula rectovaginal y colitis aguda) ${ }^{111-113}$.

\section{EN PACIENTES CON ENFERMEDAD HEMORROIDAL INTERNA Y HEMORRAGIA EN LOS QUE HA FALLADO EL TRATAMIENTO MÉDICO INICIAL SE RECOMIENDA TRATAMIENTO CON LIGADURA (DISPOSITIVO NO ENDOSCÓPICO), ESCLEROTERAPIA O FOTOCOAGULACIÓN INFRARROJA}

\footnotetext{
Recomendación fuerte, calidad de evidencia moderada (GRADE 1B)

La enfermedad hemorroidal es una causa frecuente de $\mathrm{HTDB}^{6}$. El tratamiento se dirige a eliminar los síntomas como dolor, sangrado y prolapso hemorroidal114.

La selección del tratamiento medicoquirúrgico se basa en la clasificación de Goligher, que estratifica al prolapso observado en cuatro grados ${ }^{115}$.
}

En pacientes con enfermedad hemorroidal y sangrado persistente a pesar de tratamiento médico se requiere alguna otra intervención, que puede ser desde procedimientos realizados en consultorio a cirugía (no resectiva o resectiva) ${ }^{116}$.

Los procedimientos de consultorio tienen como objetivo mejorar los síntomas mediante la disminución del tamaño o vasculatura del tejido hemorroidal, así como mejorar la fijación a la pared rectal, e incluyen: ligadura con banda elástica, escleroterapia y fotocoagulación ${ }^{117}$. Sus ventajas son bajo costo, dolor mínimo y recuperación rápida; sus desventajas incluyen mayor riesgo de recurrencia y necesidad de nuevas intervenciones ${ }^{118}$ : - La ligadura con bandas es el procedimiento más usado y eficaz, en especial cuando es realizado correctamente y en pacientes bien seleccionados. Se ha reportado una tasa de curación del 69-97\% y de recurrencia del $6.6-18 \%$ a dos años ${ }^{119}$. Su eficacia a largo plazo en comparación con otros procedimientos de consultorio es mayor, además de asociarse a menor necesidad de otras terapias y menor tasa de complicaciones; sin embargo, la tasa de dolor es mayor (RM: 2.6-14.5; $p<0.0001)^{120}$.

Una revisión de Cochrane que comparó ligadura con bandas y la hemorroidectomía convencional en pacientes con enfermedad hemorroidal grado II y III concluyó que la ligadura debería ser adoptada como el tratamiento de elección en pacientes con enfermedad grado II, ya que entre ambas técnicas no se encontraron diferencias en la recurrencia (RR: 1.07; IC 95\%: 0.94-1.21; $p>0.05$ ), pero sí menor tasa de dolor (RR: 1.94; IC 95\%: 1.62-2.33; $p<0.00001$ ) y una recuperación más rápida en la ligadura y mayor riesgo de complicaciones (RR: 6.32; IC 95\%: 1.15-34.89; $p$ < 0.05) para la hemorroidectomía. Esta última se debe reservar para pacientes para enfermedad grado III 0 aquellos con recurrencia posterior a la ligadura ${ }^{121}$.

- El fenol al $5 \%$ y el polidocanol son los agentes más ampliamente utilizados en la escleroterapia. Este tratamiento es simple, seguro y rápido, pero no tan efectivo como la ligadura con bandas; debe reservarse para pacientes con sangrado como síntoma principal, que no responden a la terapia conservadora o con un alto riesgo de hemorragia secundaria al procedimiento, como aquellos con uso de anticoagulantes, cirrosis avanzada o inmunocomprometidos. La calidad de la evidencia disponible sobre su eficacia es baja debido a la heterogeneidad en el diseño de los estudios. Un estudio reciente, con seguimiento de un año, reportó una tasa de éxito del $20 \%$ en 
enfermedad hemorroidal grado III, mientras que para la grado I la tasa se eleva hasta el $88 \% 114,122,123$.

- La fotocoagulación infrarroja es un procedimiento relativamente sencillo, sin embargo requiere varias sesiones por paquete hemorroidal y es de costo elevado comparado con otros procedimientos. Es idóneo para pacientes con enfermedad hemorroidal interna grado I a III, en quienes se ha demostrado un control del sangrado en un $78.4,51.6$ y $22.2 \%$ respectivamente durante un seguimiento a seis meses; sin embargo, la recurrencia es del $49 \%$ y hasta un $28 \%$ requieren un nuevo procedimiento. Comparado con la aplicación de ligas, las molestias y síntomas posteriores al procedimiento son menores (dolor, $16.3 \%$ y sangrado, $15.2 \%)^{114,124,125}$.

En conclusión, el éxito de los procedimientos de consultorio depende de la adecuada selección del paciente y la correcta ejecución del procedimiento. Lo anterior enfatiza la importancia de una adecuada valoración y la realización de estos por personal especializado (coloproctólogo). Los pacientes con enfermedad hemorroidal grado II o III con persistencia de sangrado a pesar de la realización de procedimientos de consultorio deben ser evaluados por un coloproctólogo, quien considerará la necesidad de tratamiento quirúrgico.

\section{EL DIAGNÓSTICO DE SÍNDROME DE ÚLCERA RECTAL SOLITARIA DEBE ESTABLECERSE CON BASE EN LAS CARACTERÍSTICAS CLÍNICAS, ENDOSCÓPICAS E HISTOPATOLÓGICAS}

\section{Recomendación fuerte, calidad de evidencia baja (GRADE 2C)}

El síndrome de úlcera rectal solitaria es un trastorno crónico, benigno y poco común, caracterizado por una combinación de síntomas, hallazgos endoscópicos y anomalías histológicas ${ }^{126}$. Es una patología de adultos jóvenes, que ocurre con mayor frecuencia en la tercera y cuarta década de la vida, con discreto predominio en las mujeres. La etiopatogenia involucra factores como trauma directo o isquemia. El prolapso de la mucosa evidente u oculta es el mecanismo patogénico subyacente más común; esto provoca congestión venosa, flujo sanguíneo deficiente, edema de la mucosa rectal, cambios inflamatorios e isquémicos y el desarrollo de úlceras en la mitad de las veces. Otros mecanismos asociados son el síndrome del perineo descendido y disinergia anorrectal.

Las características clínicas incluyen rectorragia (en el $85 \%$ ), estreñimiento (en el $75 \%$ ), prolapso rectal (en el $20 \%$ ), mucorrea, pujo, tenesmo y dolor anorrectal o en hipogastrio. La rectorragia puede ser desde sangre fresca escasa hasta una hemorragia grave que requiere transfusión ${ }^{126-129}$.

La úlcera generalmente se localiza en la pared anterior o lateral en el tercio medio del recto y la distancia desde el margen anal varía entre 3 y $10 \mathrm{~cm}$; en el $30 \%$ de los casos son múltiples y suelen tener un diámetro de entre 1 y $1.5 \mathrm{~cm}$, aunque pueden llegar hasta $4 \mathrm{~cm}$. Su aspecto varía desde cambios hiperémicos preulcerosos de la mucosa rectal hasta úlceras cubiertas por una escara blanca, gris o amarillenta. La úlcera puede ser superficial y la mucosa adyacente puede aparecer nodular o granular. En el $25 \%$ de los casos puede aparecer como una lesión polipoidea. Es importante tomar biopsia del área afectada para confirmación diagnóstica y excluir otros diagnósticos, incluyendo el cáncer ${ }^{130-132}$. Histológicamente se caracteriza por una mucosa elongada con glándulas distorsionadas. La lámina propia se observa edematosa y presenta proliferación de fibroblastos, y la capa muscular interna presenta engrosamiento.

\section{EN PACIENTES CON HEMORRAGIA DIVERTICULAR SE RECOMIENDA TRATAMIENTO ENDOSCÓPICO MECÁNICO, TÉRMICO O EN COMBINACIÓN CON INYECCIÓN}

Recomendación fuerte, calidad de evidencia moderada (GRADE 1C)

El sangrado de divertículos colónicos es la causa más común de HTDB y representa aproximadamente el $40 \%$ de los episodios de hemorragia grave. La prevalencia es del $30 \%$ en pacientes de 50 años de edad y alcanza el $60 \%$ en mayores de 80 años. Tiene una remisión espontánea hasta en el $80 \%$ de los ca$\operatorname{sos}^{17,133,134}$. Los métodos endoscópicos disponibles para su manejo incluyen la colocación de endoclips, la ligadura endoscópica, la terapia de inyección y los métodos térmicos ${ }^{135-140}$.

Tras la identificación del divertículo sangrante o con EHR se debe inyectar epinefrina en el cuello del divertículo a una dilución de 1:10,000 o de 1:20,000 en alícuotas de 1-3 ml en los cuatro cuadrantes. La inyección ayudará a detener 0 a disminuir la intensidad del sangrado para poder aplicar un método hemostático definitivo. Esta terapia combinada reporta una recurrencia de hemorragia a 30 días del 15 al $38 \%$ y después de ese periodo entre el 5 y $13 \% 47,88,140$.

Los métodos térmicos pueden ser monopolares 0 bipolares. La sonda monopolar se debe emplear con una energía de 10-15 J y la bipolar con 10-16 W en pulsos de 2-3 s; se debe aplicar una presión moderada 
sobre el tejido evitando aplicaciones múltiples o rangos altos de energía para prevenir la lesión transmural y la perforación (sobre todo en la pared más delgada del colon derecho). La recurrencia de hemorragia reportada a 30 días es del 19 al $40 \%$ y posterior a este tiempo, entre el 10 y el $40 \%{ }^{78,140}$.

La aplicación de clips endoscópicos es rápida y eficaz. Los clips deben colocarse sobre el vaso sangrante en el cuello del divertículo o afrontando las paredes de este para cerrar el orificio. Se puede emplear un capuchón para evertir el divertículo y facilitar la colocación de un clip en el domo diverticular. Diversos autores reportaron una frecuencia del 0 al $34 \%$ y consideraron que la hemorragia localizada en el colon derecho es un factor de riesgo para hemorragia refractaria ${ }^{39,35,136,141}$.

La ligadura se asocia a hemostasia inmediata en todos los casos, sin complicaciones, con una recurrencia temprana de la hemorragia hasta del $11 \%$. Cuando se vaya a utilizar ligadura, se sugiere marcar el divertículo a tratar con un clip, retirar el endoscopio y colocar el dispositivo de ligas. También se recomienda el tatuaje del sitio adyacente para su identificación en caso de recurrencia de sangrado y facilitar el tratamiento endoscópico o la intervención quirúrgica. La colocación de un endoclip puede ayudar a la identificación del sitio de sangrado en una angiografía $88,135,137,142-144$.

Considerando la evidencia disponible y su calidad, consideramos que el método mecánico es el más efectivo y que la inyección con epinefrina ayuda a mejorar la visión y facilitar la aplicación de un método definitivo.

\section{Otros métodos diagnósticos y terapéuticos en hemorragia del tubo digestivo bajo}

\section{Otras causas de hemorragia aguda de tubo digestivo bajo}

\section{LAS ÚLCERAS Y VÁRICES RECTALES O LA LESIÓN DE Dieulafoy Representan MENOS DEL $10 \%$ Del tOtal DE ETIOLOGÍAS Y SE TRATAN DE FORMA CONVENCIONAL \\ Recomendación fuerte, calidad de evidencia baja (GRADE 1C)}

Otras causas de HTDB incluyen a las úlceras rectales, las várices rectales y la lesión de Dieulafoy. Las úlceras rectales son la causa de aproximadamente el $8 \%$ de los casos de hematoquecia, sin embargo, esta cifra llega hasta el $31 \%$ en los pacientes que se encuentran en la terapia intensiva ${ }^{145,146}$.
Las várices rectales se han reportado en el 38 al 94\% de los pacientes con hipertensión portal, sin embargo solo del 0.5 al $18 \%$ desarrollan sangrados ${ }^{6,147}$. Aunque la evidencia es escasa, la inyección con cianoacrilato ha mostrado utilidad y menor recurrencia de la hemorragia cuando se compara con ligadura. En casos graves y refractarios se han empleado tratamientos dirigidos a disminuir la hipertensión portal, como el TIPS (transjugular intrahepatic portosystemic shunt) y el BRTO (balloon-occluded retrograde obliteration).

La lesión de Dieulafoy es la causa de menos del 5\% de los casos de HTDB con una mortalidad asociada del 9 al $13 \%{ }^{148}$. La eficacia del tratamiento endoscópico es similar al de otras localizaciones.

Comentario: aunque su prevalencia es baja, es importante identificar adecuadamente estas lesiones para poder ofrecer el mejor tratamiento posible. En el caso de las várices rectales debe diferenciarse correctamente de los plexos hemorroidales en pacientes con hemorragia digestiva baja.

\section{Otros métodos terapéuticos en hemorragia del tubo digestivo bajo}

\section{El tratamiento de la hemorRagia deL tUBO}

DIGESTIVO BAJO CON PLASMA ARGÓN ES UNA TÉCNICA EFECTIVA Y SEGURA

\section{Recomendación fuerte, calidad de evidencia baja (GRADE 1C)}

La frecuencia de efectos adversos de la colonoscopia terapéutica se ubica entre el 0.3 y el $1.3 \%{ }^{39}$ Los métodos y técnicas utilizadas son los mismos a los descritos en la HTDA.

La terapia con APC monopolar es un método de no contacto de fácil aplicación que puede utilizarse como terapia única o combinada ${ }^{149,150}$. Su seguridad y eficacia depende de la etiología de la hemorragia (p. ej., en pacientes con enfermedad diverticular de colon, su papel es limitado y comprometido por el elevado riesgo de perforación comparado con otros métodos endoscópicos de tipo mecánico como los clips [8 vs. $1 \%$; $p<0.05$, que parece estar asociado al grado de penetración sobre todo en una superficie delgada, como lo es el fondo de un divertículo, por lo cual en estos casos se prefieren otros métodos hemostáticos) $)^{151-154}$.

La mayor utilidad se observa en hemorragia secundaria a lesiones vasculares del tipo angiodisplasia, telangiectasias (incluso de colon derecho) y en proctopatía posrradiación en donde la eficacia 
hemostática inmediata y de erradicación de la lesión posterior a la aplicación de argón es mayor al $95 \%{ }^{155,156}$.

En pacientes con proctitis posrradiación ${ }^{157}$ el APC fue igualmente efectivo comparado con la formalina (94 vs. $100 \%$, respectivamente) en el tratamiento, logrando erradicación de las lesiones en una mediana de dos sesiones $(p=N S)$, sin que se hayan reportado diferencias en los eventos adversos entre ambos métodos.

La tasa de perforación y eficacia terapéutica del argón en ese tipo de lesiones se ha reportado del $0.05 \%$ y superior al $95 \%$ respectivamente en otros estudios ${ }^{158}$. Los eventos adversos reportados incluyen dolor rectal (7\%) y formación de úlceras (12\%), las cuales no generan complicaciones mayores ${ }^{107}$. La aplicación de APC en lesiones vasculares ha demostrado mejorar los niveles de $\mathrm{Hb}$, y reducir el número de transfusiones y los días de estancia intrahospitalaria ${ }^{159}$.

Se recomienda la siguiente configuración de parámetros del argón en el tratamiento de lesiones vasculares: potencia entre 20 y $60 \mathrm{~W}$, un flujo de $1.5-2 \mathrm{l} / \mathrm{min}$ y pulsos de $0.5-2 \mathrm{~s}$; en lesiones de colon derecho o lesiones grandes $(>10 \mathrm{~mm})$ se deben utilizar potencias de entre 20 y $30 \mathrm{~W}$, además se recomienda realizar un colchón submucoso con solución salina para disminuir el riesgo de perforación (3-5\%) ${ }^{160}$.

La información sobre el uso de APC en el tratamiento de hemorragia pospolipectomía es limitado, con tasas de respuesta entre el 65 y el $70 \%$, por lo que se prefieren otros métodos como el clip hemostático o la combinación de métodos de inyección y térmicos de contacto $^{161}$.

Como ya se comentó, su papel en otras causas de hemorragia es solo como puente antes de ofrecer un tratamiento definitivo, considerándose un método seguro (<3\% perforación) y efectivo $(>80 \%)^{162}$.

En casos de recurrencia de hemorragia de diferentes etimologías, especialmente la diverticular, no se considera un método de primera elección, puesto que son superiores otros métodos como los clips o la terapia bipolar (eficacia superior al $85 \%)^{163}$. En angiodisplasias es un método seguro (perforación $<2 \%$ ) y efectivo $(>90 \%)$, pero sin diferencia con lo encontrado al compararse con otros métodos de tratamiento endoscópico ${ }^{164}$.

Comentario: en nuestro medio, la utilización actual del argón es principalmente en lesiones vasculares, generalmente a potencias bajas (20-30 W), y si se trabaja en colon derecho se recomienda realizar un colchón de seguridad con solución salina con o sin combinación con azul de metileno.

\section{EN RECURRENCIA DE HEMORRAGIA PUEDE} CONSIDERARSE REALIZAR UNA NUEVA COLONOSCOPIA

\section{Recomendación fuerte, calidad de evidencia baja (GRADE 1C)}

La recurrencia de HTDB se define como temprana cuando ocurre durante la estancia intrahospitalaria asociada a la hemorragia; su frecuencia ha sido reportada de hasta un $22 \%$ de los casos con HTDB confirmados mediante colonoscopia de urgencia. Cuando la recurrencia de HTDB aparece una vez egresado el paciente, se define como tardía y se presenta hasta en un $16 \%$ de los $\operatorname{cas}^{44,47,165}$. Entre los factores asociados a la recurrencia destacan las comorbilidades del paciente, el uso de anticoagulantes y AINE, entre otros $^{136}$. La colonoscopia logra documentar el origen de la recurrencia hasta en el $14 \%$ de los casos y logra realizar un cambio en el diagnóstico inicial hasta en el $20 \%$ de los pacientes, principalmente en enfermedad diverticular de colon $^{162}$. En un paciente con recurrencia de HTDB y que se encuentra hemodinámicamente estable está indicado realizar una colonoscopia de forma inicial.

En la primera revisión puede haber casos en donde los pacientes pueden tener restos hemáticos combinado con una mala preparación intestinal, por lo cual está indicado realizar o mejorar la preparación intestinal para realizar una adecuada evaluación.

Comentario: nuestra recomendación en una recurrencia de hemorragia en un paciente hemodinámicamente estable es realizar una colonoscopia con una preparación intestinal adecuada, considerando que con este abordaje aumentaremos nuestra capacidad diagnóstica en un $20 \%$.

LOS POLVOS HEMOSTÁTICOS PUEDEN SER UTILIZADOS COMO TERAPIA DE RESCATE EN HEMORRAGIA AGUDA DE TUBO DIGESTIVO BAJO

\section{Recomendación fuerte, calidad de evidencia baja (GRADE 1C)}

La HTDB continúa siendo un reto diagnóstico y terapéutico; en ocasiones se requieren medidas de salvamento para su tratamiento. Actualmente se han desarrollado polvos hemostáticos, por ejemplo, Hemospray ${ }^{\circledR}$ (TC-325), Endoclot ${ }^{\circledR}$ (AMP) y Ankafred Blood Stopper $^{\circledR}$, de los cuales solo los dos primeros están disponibles en México.

Un metaanálisis que evaluó la utilidad de los polvos hemostáticos como terapia de rescate en diversas etiologías de HTDB (p. ej., hemorragia posterior a 
polipectomía, tumores colónicos, divertículos, malformaciones arteriovenosas, proctitis posrradiación, colitis isquémica y hemorragia en anastomosis quirúrgicas) concluyó que se logró hemostasia exitosa en el 88 al 100\% de los casos, con una recurrencia del 3 al $13 \%$, excepto en la proctitis posrradiación, donde la recurrencia fue hasta del $77 \%{ }^{166}$.

En un estudio prospectivo observacional de 70 pacientes con HTD, de los cuales 12 fueron de tubo digestivo bajo, la hemostasia exitosa utilizando AMP fue del $83 \%$, con solo dos casos de recurrencia ${ }^{167}$. En otro estudio retrospectivo de 60 pacientes (11 con HTDB) en los que se utilizó TC-325 como tratamiento de hemorragia activa de tubo digestivo, se logró hemostasia en el $100 \%$ de los casos sin que se reportaran recurrencias $^{92}$. El Ankafred Blood Stopper ${ }^{\circledR}$, por su parte, ha sido utilizado en 19 casos logrando una hemostasia del $100 \%$, sin presentar recurrencia de hemorragia ${ }^{168}$.

Comentario: los polvos hemostáticos son un método seguro y eficaz en la hemostasia de HTDB, sin que existan reportes hasta el momento de la elaboración de esta guía de eventos secundarios derivados de su utilización. Aunque la evidencia existente es poca, su uso ha demostrado gran eficacia en terapias de rescate en la HATDB. La recurrencia de hemorragia posterior a su aplicación es baja y cuando se presenta no suele ser grave, por lo que se cuenta con el tiempo necesario para estabilizar al paciente y realizar una nueva colonoscopia en condiciones adecuadas.

\section{EL DIAGNÓSTICO Y TRATAMIENTO RADIOLÓGICO DE LA HEMORRAGIA AGUDA DE TUBO DIGESTIVO BAJO SE RESERVA A PACIENTES CON HEMORRAGIA ACTIVA E INESTABILIDAD HEMODINÁMICA}

\section{Recomendación fuerte, calidad de evidencia alta (GRADE 1A)}

El objetivo de la valoración radiológica de la HTDB es proporcionar información acerca de la actividad e intensidad de la hemorragia, así como su localización ${ }^{169}$. El uso y elección de estos métodos dependerá de la intensidad y gravedad de la hemorragia.

La tomografía multicorte (angiotomografía o angio-TC) es una herramienta versátil, sensible (86\%) y específica (95\%) capaz de detectar la presencia de hemorragia activa cuando esta se presenta con una tasa o velocidad de sangrado de tan solo $0.4 \mathrm{ml} / \mathrm{min}$, además de localizar la región anatómica afectada. Su utilidad en la HTDB se limita a un método diagnóstico en aquellos casos en los que no se localiza la fuente de sangrado por métodos endoscópicos y que están hemodinámicamente estables, pues esto último permite realizar el estudio adecuadamente y con seguridad ${ }^{170,171}$.

En pacientes con hemorragia masiva (que requieren más de cinco unidades de sangre para transfusión en $24 \mathrm{~h}$ ), inestabilidad hemodinámica, en quienes la colonoscopia puede ser técnicamente difícil y el tiempo de preparación resulta una limitante para su realización, la angiografía por sustracción digital se considera la primera opción diagnóstica y terapéutica ${ }^{171-173}$. No debe utilizarse con fines diagnósticos o terapéuticos en pacientes hemodinámicamente estables, ya que es invasiva y se asocia a complicaciones potencialmente graves, como isquemia intestinal $(23 \%)$, estenosis de colon $(23 \%)$ y perforación (5\%); también tiene una disponibilidad limitada y requiere de personal entrenado para su realización e interpretación. Sin embargo, tiene la gran ventaja de poder ofrecer un tratamiento definitivo, ya sea por medios químicos (alcohol polivinílico) o físicos (coils, microcoils), con una alta eficacia hemostática que llega a ser de hasta del $100 \%$ en casos de embolización supraselectiva ${ }^{171-175}$.

Comentario: en nuestro medio la disponibilidad de radiólogos intervencionistas es limitada; los hospitales que cuentan con este recurso son pocos y generalmente son centros de referencia, sin embargo, la evidencia apoya una recomendación sólida considerando los beneficios en este grupo de pacientes. De forma que en un paciente con hemorragia digestiva baja hemodinámicamente inestable se debe consultar de primera intención al cirujano de guardia, posteriormente realizar una angiografía supraselectiva diagnóstica y terapéutica y si esta falla o no está disponible, se deberá optar por tratamiento quirúrgico.

\section{DEBIDO A LA GRAN VARIEDAD DE TRATAMIENTOS} ENDOSCÓPICOS Y RADIOLÓGICOS, LA CIRUGÍA SE CONSIDERA EL ÚLTIMO RECURSO EN EL TRATAMIENTO DE LA HEMORRAGIA AGUDA DE TUBO DIGESTIVO BAJO

\section{Recomendación débil, calidad de evidencia baja (GRADE 2C)}

La evidencia respecto al papel de la cirugía en la HATDB es pobre y se deriva principalmente de revisiones retrospectivas pequeñas.

Un estudio prospectivo multicéntrico en el Reino Unido que incluyó 2,528 pacientes con HTDB observó que solo $54 / 2,528(2.1 \%)$ requirieron tratamiento endoscópico y solo $6 / 2,528(0.02 \%)$ fueron sometidos a laparotomía exploratoria por hemorragia activa no controlada por otros métodos, de los cuales $3 / 6(50 \%)$ murieron. Su conclusión fue que la cirugía debería ser 


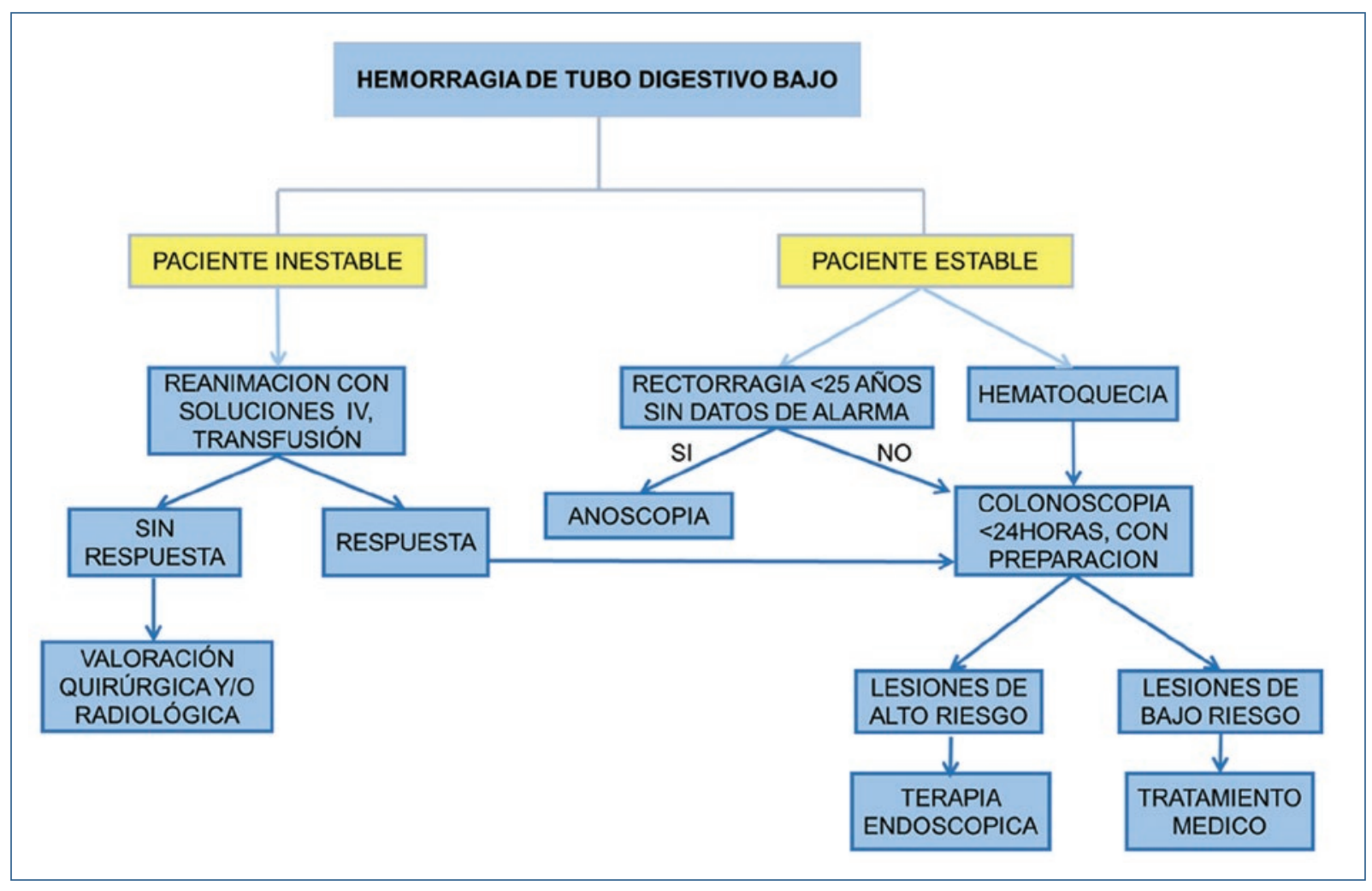

Figura 1. Algoritmo de manejo.

el último recurso después de que el manejo por colonoscopia y radiología intervencionista ha fallado ${ }^{176}$.

Extraído de una revisión sistemática de estudios publicados entre el año 2000 y $2015^{44}$, sobre pacientes que recibieron tratamiento endoscópico por HTDB, en el estudio de Jensen, et al., que evaluó a 48 pacientes con HTDB ${ }^{56}$, en 14/48 (29\%) no se documentó un sitio claro de hemorragia y en seis de ellos se realizó hemicolectomía basando la decisión solo en la presencia de EHR, sin que ninguno de ellos hubiera recibido tratamiento endoscópico previo. Se concluyó que en los pacientes tratados previamente de forma endoscópica existe una disminución en el riesgo de requerir cirugía (RR: 0.14; IC 95\%: 0.02-0.88), menor riesgo de recurrencia de hemorragia (RR: 0.10; IC 95\%: 0.020.51) y de transfusión (RR: 0.10; IC 95\%: 0.02-0.51), por lo que se sugiere el tratamiento endoscópico con inyección de epinefrina o coagulación bipolar o ambos, pues previene la recurrencia de hemorragia y disminuye la necesidad de tratamiento quirúrgico ${ }^{5,7}$.

Comentario: en general, la cirugía en la HATDB debe considerarse solo cuando otras opciones terapéuticas han fallado o han tenido complicaciones. Siempre debe tomarse en cuenta la severidad y origen de la hemorragia, así como las comorbilidades del paciente, evitando en la medida de lo posible medidas de emergencia como una colectomía segmentaria a ciegas.

\section{Conclusiones}

La presente guía de la AMEG sobre abordaje y manejo endoscópico de la hemorragia de tubo digestivo bajo es el resultado de un análisis multidisciplinario basado en la evidencia disponible hasta el momento de su elaboración y en la opinión de especialistas expertos. Esta guía tiene la intención de proveer recomendaciones en puntos clave por parte de los participantes que ayuden al adecuado diagnóstico y tratamiento a los médicos relacionados con el cuidado de estos pacientes. Es importante recordar que la presente guía debe interpretarse e implementarse basándose en cada caso individual y en conjunto con un equipo multidisciplinario. El presente documento estará sujeto a modificaciones previas a su publicación de acuerdo con las correcciones finales de los editores de la revista mexicana de endoscopia. Una síntesis de las guías y un algoritmo resultado de las mismas se muestran en la Tabla 8 y la Figura 1. 
Tabla 8. Síntesis de las recomendaciones

I. Generalidades de la HTDB

1. Definición

1.1. La HTDB se define como la originada distal a la válvula ileocecal; su presentación clínica puede ser con hematoquecia, rectorragia o melena. GRADE $1 \mathrm{C}$

2. Epidemiología

2.1. La HATDB representa hasta el $25 \%$ de las hemorragias gastrointestinales. La frecuencia etiológica varía de acuerdo con el grupo etario. GRADE $1 \mathrm{C}$

3. Evaluación inicial y estratificación de riesgo del paciente con HATDB

3.1. La evaluación inicial del paciente con HATDB debe incluir historia clínica rápida, examen físico y estudios de laboratorio para determinar la severidad de la hemorragia, etiología y posible localización de esta. GRADE 1C

3.2. La estratificación de riesgo (alto o bajo) permite predecir mortalidad, riesgo de recurrencia de la hemorragia, necesidad de intervención endoscópica urgente e ingreso a cuidados intensivos. GRADE 1B

4. Tratamiento inicial de la HATDB: restitución de volumen, transfusión, corrección de la coagulopatía

4.1. El primer paso en el manejo de la HATDB es evaluar el estado hemodinámico y la reanimación intensiva antes de realizar el procedimiento endoscópico. GRADE 1B

4.2. En la HATDB se recomienda la estrategia de transfusión restrictiva. GRADE 1B

4.3. La coagulopatía moderada (INR < 2.5) no contraindica la endoscopia diagnóstica ni terapéutica. GRADE 1B

II. HTDB de origen en colon

1. Papel de la colonosocopia en la HATDB

1.1. La colonoscopia es el método diagnóstico de elección en pacientes con HATDB con sospecha de origen en colon. GRADE 1B

2. Colonoscopia temprana en la HATDB

2.1. En pacientes hemodinámicamente estables con HATDB persistente se recomienda la colonoscopia temprana (en las primeras $24 \mathrm{~h}$ ), posterior a una preparación del colon. GRADE 2B

3. Características de la preparación de colon en la HATDB

3.1. La preparación enérgica del colon incrementa el rendimiento diagnóstico y terapéutico en la HTDB. GRADE 1B

4. Características del tratamiento endoscópico de la HATDB originada en colon

4.1. La selección del tratamiento de la HATDB dependerá de los hallazgos endoscópicos, los cuales pueden clasificarse en de alto y bajo riesgo. GRADE $1 \mathrm{C}$

4.2. Debe llevarse a cabo tratamiento endoscópico hemostático en lesiones con estigmas de alto riesgo o hemorragia activa. GRADE 1C

4.3. El empleo de inyección de adrenalina puede ser útil para lograr hemostasia inicial o para mejorar la visualización de las lesiones sangrantes. GRADE $1 \mathrm{~A}$

4.4. En hemorragia pospolipectomía las terapias de inyección, térmicas y mecánicas son útiles para el tratamiento. GRADE 1A

4.5. La colitis isquémica, la colitis infecciosa, la neoplasia colorrectal y la Ell se asocian con hemorragia difusa; sin embargo, la pertinencia del manejo endoscópico debe individualizarse. GRADE 1C

III. Hemorragia de tubo digestivo de origen anorrectal

1. Exploración física y estudios complementarios

1.1. La exploración anorrectal y la anoscopia están indicadas en presencia de rectorragia. GRADE 1B

1.2. Los estudios complementarios de la exploración anorrectal incluyen la rectosigmoidoscopia flexible, la rectosimoigdoscopia rígida y la colonoscopia. GRADE 1B

2. Etiologías de la HTDB de origen anorrectal

2.1. Para el tratamiento endoscópico de la hemorragia del tubo digestivo bajo por proctopatía por radiación se recomienda el uso de APC. GRADE 1B

2.2. Otras alternativas de tratamiento endoscópico de la HTDB secundaria a proctopatía por radiación incluyen la aplicación tópica de formalina (2-10\%), la ablación por radiofrecuencia, la crioterapia y la terapia con láser. GRADE 2C

2.3. En pacientes con enfermedad hemorroidal interna con sangrado en los que ha fallado el tratamiento médico inicial se recomienda tratamiento con ligadura (dispositivo no endoscópico), escleroterapia o fotocoagulación infrarroja. GRADE 1B

2.4. El diagnóstico de síndrome de úlcera rectal solitaria debe establecerse con base en las características clínicas, endoscópicas e histopatológicas. GRADE $2 \mathrm{C}$

2.5. En pacientes con hemorragia diverticular se recomienda tratamiento endoscópico mecánico, térmico o en combinación con inyección. GRADE 1C

IV. Otros métodos diagnósticos y terapéuticos en la HTDB

1. Otras causas de HATDB

1.1. Las úlceras y várices rectales o la lesión de Dieulafoy representan menos del 10\% del total de etiologías y se tratan de forma convencional. GRADE $1 \mathrm{C}$

2. Otros métodos terapéuticos en la HTDB

2.1. El tratamiento de la HTDB con APC es una técnica efectiva y segura incluso en colon derecho. GRADE 1C

2.2. En recurrencia de hemorragia puede considerarse realizar una nueva colonoscopia. GRADE 1C

2.3. Los polvos hemostáticos pueden ser utilizados como terapia de rescate en la HTDB. GRADE 1C

2.4. El diagnóstico y tratamiento radiológico de la HATDB se reserva a pacientes con hemorragia activa e inestabilidad hemodinámica. GRADE 1 A

2.5. Debido a la gran variedad de tratamientos endoscópicos y radiológicos, la cirugía debe ser considerada como el último recurso en el tratamiento de la HTDB. GRADE $2 \mathrm{C}$

APC: plasma argón; GRADE: Grading of Recommendations, Assessment, Development and Evaluation; HATDB: hemorragia aguda de tubo digestivo bajo; HTDB: hemorragia de tubo digestivo bajo; INR:. 


\section{Declaración de conflictos de interés}

Los siguientes participantes declaran tener algún conflicto de interés o relación financiera: Angélica Hernández Guerrero es conferencista de Olympus Inc. y Boston Scientific; Alejandra Noble Lugo es conferencista de Asofarma, Menarini, Takeda, AlfaSigma y AstraZeneca; José Luis Rocha Ramírez es conferencista de Abbvie, Janssen, Pfizer y Ferring.

Los participantes no enlistados anteriormente declaran no tener relaciones financieras y/o potenciales conflictos de interés.

\section{Bibliografía}

1. Fink A, Kosecoff J, Chassin M, Brook RH. Consensus methods: characteristics and guidelines for use. Am J Public Health. 1984;74:979-83.

2. Grading Tutorial. Grading Recommendations in UpToDate [Internet]. Wolters Kluwer. UpToDate [consultado en línea 15 de marzo 2017]. Disponible en: http://www.uptodate.com/home/grading-tutorial.

3. Guyatt GH, Oxman AD, Vist GE, Kunz R, Falck-Ytter Y, Alonso-Coello P, et al. GRADE: an emerging consensus on rating quality of evidence and strength of recommendations. BMJ. 2008;336:924-6.

4. Blanco-Vela $\mathrm{Cl}$, Peláez-Luna M, Hernández-Guerrero. Clinical guidelines for diagnostic and management of non-variceal gastrointestinal bleeding. Endoscopia. 2017;29(3):173-95.

5. Strate L, Gralnek I. ACG Clinical Guideline: Management of patients whit acute lower gastrointestinal bleeding. Am J Gastroenterol. 2016;111(4):459474.

6. Barnert $\mathrm{J}$, Messmann $\mathrm{H}$. Diagnosis and management of lower gastrointestinal bleeding. Nat Rev Gastroenterol Hepatol. 2009;6:637-46.

7. Diamantopoulou G, Konstantakis C, Kottorou K, Skroubis G, Theocharis G, Theopistos V, et al. Acute lower gastrointestinal bleeding: characteristics and clinical outcome of patients treated with an intensive protocol. Gastroenterol Res. 2017;10(6):352-8.

8. Gralnek I, Neeman Z, Strate L. Acute lower gastrointestinal bleeding. N Engl J Med. 2017;376:1054-63.

9. Saperas E. Lower gastrointestinal bleeding: the great unknown. Gastroenterol Hepatol. 2007;30:93-100.

10. Aoki T, Nagata N, Niikura R, Shimbo T, Tanaka S, Sekine K, et al. Recurrence and mortality among patients hospitalized for acute lower gastrointestinal bleeding. Clin Gastroenterol Hepatol. 2015;13:488-94.e1.

11. Ghassemi KA, Jensen DM. Lower GI bleeding: epidemiology and management. Curr Gastroenterol Rep. 2013;15:333.

12. Hreinsson JP, Gumundsson S, Kalaitzakis E, Björnsson ES. Lower gastrointestinal bleeding: incidence, etiology, and outcomes in a population-based setting. Eur J Gastroenterol Hepatol. 2013;25:37-43.

13. Merino O, Bustamante J, Frenández-Ramos JR, GH Continuada. 2003; 2:49-53.

14. Wilcox CM, Alexander LN, Cotsonis G. A prospective characterization of upper gastrointestinal hemorrhage presenting with hematochezia. Am J Gastroenterol. 1997:92(2):231-5.

15. Sittichanbuncha Y, Senasu S, Thongkrau T, Keeratikasikorn C, Sawanyawisuth K. How to differentiate sites of gastrointestinal bleeding in patients with hematochezia by using clinical factors? Gastroenterol Res Pract. 2013;2013:265076.

16. Tomizawa M, Shinozaki F, Hasegawa R, Shirai Y, Motoyoshi Y, Sugiyama T, et al. Laboratory test variables useful for distinguishing upper from lower gastrointestinal bleeding. World J Gastroenterol. 2015;21(20):6246-51.

17. Longstreth GF. Epidemiology and outcome of patients hospitalized with acute lower gastrointestinal hemorrhage: a population-based study. Am J Gastroenterol. 1997:92:419-24.

18. Lanas A, Garcia-Rodriguez LA, Polo-Tomas M, Ponce M, Alonso-Abreu I, Perez-Aisa MA, et al. Time trends and impact of upper and lower gastrointestinal bleeding and perforation in clinical practice. Am J Gastroenterol. 2009;104:1633-41.

19. Strate LL, Orav EJ, Syngal S. Early predictors of severity in acute lower intestinal tract bleeding. Arch Intern Med. 2003;163(7):838-43.

20. Strate LL, Saltzman JR, Ookubo R, Mutinga ML, Syngal S. Validation of a clinical prediction rule for severe acute lower intestinal bleeding. Am J Gastroenterol. 2005;100(8):1821-7.

21. Baradian R, Ramdhaney S, Chapalamadugu R, Skoczylas L, Wang K Rivilis $S$, et al. Early intensive resuscitation of patients with upper gastrointestinal bleeding decreases mortality. Am J Gastroenterol. 2004;99:619-22.
22. Semler M, Rice T. Saline is not the first choice for crystalloid resuscitation fluids. Crit Care Med. 2016;44:1541-4

23. Wang J, Bao YX, Bai M, Zhang YG, Xu WD, Qi XS. Restrictive vs. liberal transfusion for upper gastrointestinal bleeding: a meta-analysis of randomized controlled trials. World J Gastroenterol. 2013;19:6919-27.

24. Marik PE, Corwin HL. Efficacy of red blood cell transfusion in the criticaIly ill: a systematic review of the literature. Crit Care Med. 2008;36:2667-74.

25. Gregersen M, Damsgaard E. Comparison of liberal and restrictive blood transfusion: current insights into clinical outcomes. Inter J Clin Transf Med. 2016;4:99-108.

26. Hearnshaw SA, Logan RF, Palmer KR, Card TR, Travis SP, Murphy MF. Outcomes following early red blood cell transfusion in acute upper gastrointestinal bleeding. Aliment Pharmacol Ther. 2010;32:215-24.

27. Alzoubaidi D, Lovat LB, Haidry R. Management of non variceal upper gastrointestinal bleeding: where are we in 2018. Frontline Gastroenterol. 2018;10(1):1-8.

28. Odutayo A, Desborough M. Trivella M, Stanley AJ, Dorée C, Collins GS, et al. Restrictive versus liberal blood transfusion for gastrointestinal bleeding: a systematic review and meta-analysis of randomised controlled trials. Lancet Gastroenterol Hepatol. 2017;2:354-60.

29. Villanueva $\mathrm{C}$, Colomo A, Bosch $\mathrm{A}$. Transfusion for acute upper gastrointestinal bleeding. N Engl J Med. 2013;368:1362-3

30. Zullo A, Hassan C, Radaelli. Gastrointestinal endoscopy in patients on anticoagulant therapy and antiplatelet agents. Ann Gastroenterol. 2017:30:7-14

31. Radaelli F, Dentali F, Repici A, Amato A, Paggi S, Rondonotti E, et al. Management of anticoagulation in patients with acute gastrointestinal bleeding. Dig Liver Dis. 2015;47:621-7.

32. Holster IL, Valknoff VE, Kulpers EJ, Tjwa ETTL. New oral anticoagulants increase risk for gastrointestinal bleeding: a systematic review and meta-analysis. Gastroenterology. 2013;145:105-12.

33. Nagata N, Yasunaga H, Matsui H, Fushimi K, Watanabe K, Akiyama J, et al. Therapeutic endoscopy-related Gl bleeding and thromboembolic events in patients using warfarin or direct oral anticoagulants: result from a large nationwide detabase analysis. Gut. 2018;67(10):1805-12.

34. Choudari CP, Rajgopal C, Palmer KR. Acute gastrointestinal haemorrhage in anticoagulated patients: diagnoses and response to endoscopic treatment. Gut. 1994;35:464-6.

35. Lichtenstein DR. When to stop, how to reverse and when to restart antithrombotic drugs periendoscopially in nonvariceal upper gastrointestinal bleeding. Techniques in Gastrointestinal Endoscopy. 2016;18:177-186.

36. Quayed E, Gaurav D, Nanchal RS. Lower gastrointestinal hemorrhage. Crit Care Clin. 2016;32:241-54

37. Lhewa DY, Strate LL. Pros and cons of colonoscopy in management of acute lower gastrointestinal bleeding. World J Gastroenterol. 2012; 18:1185-90.

38. ASGE Standards of Practice Committee, Pasha SF, Shergill A, Acosta RD, Chandrasekhara V, Chathadi KV, Early D, et al. The role of endoscopy in the patient with lower GI bleeding. Gastrointest Endosc. 2014;79:875-85

39. Strate LL, Naumann CR. The role of colonoscopy and radiological procedures in the management of acute lower gastrointestinal bleeding. Clin Gastroenterol Hepatol. 2010;8:333-43.

40. Sugimoto S, Mizukami T. Diagnostic and therapeutic applications of water-immersion colonoscopy. World J Gastroenterol. 2015;21:6451-9.

41. Nagata N, Niikura R, Sakurai T, Shimbo T, Aoki T, Moriyasu S, et al. Safety and effectiveness of early colonoscopy in management of acute lower gastrointestinal bleeding on the basis of propensity score matching analysis. Clin Gastroenterol Hepatol. 2016;14:558-64.

42. Kouanda AM, Somsouk M, Sewel JL, Day LW. Urgent colonoscopy in patients with lower GI bleeding: a systematic review and meta-analysis. Gastrointest Endosc. 2017;86:107-17.

43. Correa P, Teixeira C, Zago RR, Rossini G, Paccos JL, Popoutchi P, et al. Usefulness of early colonoscopy in the diagnosis and treatment of moderate or severe lower gastrointestinal bleeding. J Coloproctol. 2017;37:25-30.

44. Oakland K, Isherwood J, Lahiff C, Goldsmith P, Desborough M, Colman KS, et al. Diagnostic and treatment modalities for acute lower gastrointestinal bleeding: a systematic review. Endosc Int Open. 2017; 5:E959-67.

45. Sengupta N, Tapper EB, Feuerstein JD. Early versus delayed colonoscopy in hospitalized patients with lower gastrointestinal bleeding. A meta-analysis. J Clin Gastroenterol. 2017;51:352-9.

46. Strate LL, Syngal S. Timing of colonoscopy: Impact on lenght of hospital stay in patients with acute lower intestinal bleeding. Am J Gastroenterol. 2003;98:317-22

47. Green BT, Rockey DC, Portwood G, Tarnasky PR, Guarisco S Branch MS, et al. Urgent colonoscopy for evaluation and management of acute lower gastrointestinal hemorrhage: A randomized controlled trial. Am J Gastroenterol. 2005;100:2395-402.

48. Roshan Afshar I, Sadr MS, Strate LL, Martel M, Menard C, Barkun AN. The role of early colonoscopy in patients presenting with acute lower gastrointestinal bleeding: a systematic review and meta-analysis. Ther Adv Gastroenterol. 2018;11:1-15. 
49. Nigam N, Patel P, Sengupta N. Outcomes of early versus delayed colonoscopy in lower gastrointestinal bleeding using a hospital administrative database. J Clin Gastroenterol. 2018;52(8):721-5.

50. Navaneethan U, Njei B, Venkatesh PGK, Sanaka MR. Timing of colonoscopy and outcomes in patients with lower GI bleeding: a nationwide population-based study. Gastrointest Endosc. 2014;79:297-306.

51. Albeldawi M, Ha D, Mehta P, Lopez R, Jang S, Sanaka MR, et al. Utility of urgent colonoscopy in acute lower gastro-intestinal bleeding: a single-center experience. Gastroenterology Report. 2014;2:300-5.

52. Tada M, Shimizu S, Kawai K. Emergency colonoscopy for the diagnosis of lower intestinal bleeding. Gastroenterol Jpn. 1991;26(Suppl 3):121-4.

53. Ohyama T, Sakurai Y, Ito M, Daito K, Sezai S, Sato Y. Analysis of urgen colonoscopy for lower gastrointestinal tract bleeding. Digestion. 2000;61:189-92.

54. Niikura R, Nagata N, Shimbo T, Sakurai T, Aoki T, Moriyasu S, et al. Adverse events during bowel preparation and colonoscopy in patients with acute lower gastrointestinal bleeding compared with elective non-gastrointestinal bleeding. PLoS One. 2015;10:e0138000.

55. Jensen DM, Machicado GA. Diagnosis and treatment of severe hematochezia. The rol of urgent colonoscopy after purge. Gastroenterology. 1988;95:1569-74.

56. Jensen DM, Machicado GA, Jutabha R, Kovacs TO. Urgent colonoscopy for the diagnosis and treatment of severe diverticular hemorrhage. $N$ Engl J Med. 2000;342:78-82

57. Ron-Tal Fisher O, Gralnek IM, Eisen GM, Williams JL, Holub JL. Endoscopic hemostasis is rarely used for hematochezia: a population-based study from the Clinical Outcomes Research Initiative National Endoscopic Database. Gastrointest Endosc. 2014;79:317-25.

58. Sonnenberg A. Management of delayed postpolypectomy bleeding: a decision analysis. Am J Gastroenterol. 2012;107(3):339-42.

59. Binmoeller KF, Thonke F, Soehendra N. Endoscopic hemoclip treatment for gastrointestinal bleeding. Endoscopy. 1993;25(2):167-70.

60. Prabhu NC, Wong KSL. Achieving hemostasis and the risks associated with therapy. Gastrointest Endoscopy Clin N Am. 2015;25:123-45.

61. Selvi TS, Raju GS. Management of polypectomy complications. Gastrointest Endoscopy Clin N Am. 2015;25:335-57.

62. Ko CW, Dominitz JA. Complications of colonoscopy: magnitude and management. Gastrointest Endosc Clin N Am. 2010;20:659-71.

63. Kim HS, Kim TI, Kim WH, Kim YH, Kim HJ, Yang SK, et al. Risk factors for immediate postpolypectomy bleeding of the colon: a multicenter study. Am J Gastroenterol. 2006;101:1333-41.

64. Watabe H, Yamaji Y, Okamoto M, Kondo S, Ohta M, Ikenoue T, et al. Risk assessment for delayed hemorrhagic complication of colonic polypectomy: polyp-related factors and patient related factors. Gastrointest Endosc. 2006;64:73-8.

65. Consolo P, Luigiano C, Strangio G, Scaffidi MG, Giacobbe G, Di Giuseppe G, et al. Efficacy, risk factors and complications of endoscopic polypectomy: ten year experience at a single center. World $J$ Gastroenterol. 2008;14:2364-9.

66. Buddingh KT, Herngreen T, Haringsma J, van der Zwet WC, Vleggaar FP, Breumelhof $\mathrm{R}$, et al. Location in the right hemi-colon is an independent risk factor for delayed post-polypectomy hemorrhage: a multi-center case-control study. Am J Gastroenterol. 2011:106:1119-24.

67. Sawhney MS, Salfiti N, Nelson DB, Lederle FA, Bond JH. Risk factors for severe delayed postpolypectomy bleeding. Endoscopy. 2008:40:115-9.

68. Heldwein W, Dollhopf M, Rosch T, Meining A, Schmidtsdorff G, Hasford J et al.; Munich Gastroenterology Group. The Munich Polypectomy Study (MUPS): prospective analysis of complications and risk factors in 4000 colonic snare polypectomies. Endoscopy. 2005;37:1116-22.

69. Metz AJ, Bourke MJ, Moss A, Williams SJ, Swan MP, Byth K. Factors that predict bleeding following endoscopic mucosal resection of large colonic lesions. Endoscopy. 2011;43:506-11.

70. Moss A, Nalankilli K. Standardisation of polypectomy technique. Best Pract Res Clin Gastroenterol. 2017;31:447-53

71. Dobrowolski S, Dobosz M, Babicki A, Głowacki J, Nałecz A. Blood supply of colorectal polyp correlates with risk of bleeding after colonoscopic polypectomy. Gastrointest Endosc. 2006;63:1004-9.

72. Gimeno-Garcia AZ, de Ganzo ZA, Sosa AJ, Pérez DN, Quintero E. Incidence and predictors of postpolypectomy bleeding in colorectal polyps larger than $10 \mathrm{~mm}$. Eur J Gastroenterol Hepatol. 2012;24:520-6.

73. Paspatis GA, Paraskeva K, Theodoropoulou A, Mathou N, Vardas E, Oustamanolakis $P$, et al. A prospective, randomized comparison of adrenaline injection in combination with detachable snare versus adrenaline injection alone in the prevention of postpolypectomy bleeding in large colonic polyps. Am J Gastroenterol. 2006;101:2805-9.

74. Kouklakis G, Mpoumponaris A, Gatopoulou A, Efraimidou E, Manolas K Lirantzopoulos N. Endoscopic resection of large pedunculated colonic polyps and risk of postpolypectomy bleeding with adrenaline injection versus endoloop and hemoclip: a prospective, randomized study. Surg Endosc. 2009:23:2732-7.

75. Li LY, Liu QS, Li L, Cao YJ, Yuan Q, Liang SW, et al. A meta-analysis and systematic review of prophylactic endoscopic treatments for postpolypectomy bleeding. Int J Colorectal Dis. 2011;26:709-19.
76. Hong SP. How do I manage post-polypectomy bleeding? Clin Endosc 2012;45:282-4.

77. Coumaros D, Tsesmeli N. Active gastrointestinal bleeding: use of hemostatic forceps beyond endoscopic submucosal dissection. World J Gastroenterol. 2010;16:2061-4.

78. ASGE Technology Committee, Conway JD, Adler DG, Diehl DL, Farraye FA, Kantsevoy SV, Kaul V, et al. Endoscopic hemostatic devices. Gastrointest Endosc. 2009;69:987-96.

79. Alcaide N, Peñas-Herrero I, Sancho-del-Val L, Ruiz-Zorrilla R, Barrio J, Pérez-Miranda M. Ovesco system for treatment of postpolypectomy bleeding after failure of conventional treatment. Rev Esp Enferm Dig. 2014:106:55-8.

80. Singhal S, Changela K, Papafragkakis H, Anand S, Krishnaiah M, Duddempudi S. Over the scope clip: technique and expanding clinical applications. J Clin Gastroenterol. 2013;47:749-56.

81. Gordy SD, Rhee P, Schreiber MA. Military applications of novel hemostatic devices. Expert Rev Med Devices. 2011;8:41-7.

82. Barkun AN, Moosayi S, Martel M. Topical hemostatic agents: a systematic review with particular emphasis on endoscopic application in Gl bleeding. Gastrointest Endosc. 2013;7:692-700.

83. Park CH, Jung YS, Nam E, Eun CS, Park DI, Han DS. Comparison of efficacy of prophylactic endoscopic therapies for postpolypectomy bleeding in the colorectum: A systematic review and network meta-analysis. AM J Gastroenterol. 2016;111:1230-43.

84. Strate LL, Ayanian JZ, Kotler G, Syngal S. Risk factors for mortality in lower intestinal bleeding. Clin Gastroenterol Hepatol. 2008:6:1004-10.

85. Pardi DS, Loftus EV Jr, Tremaine WJ, Sandborn WJ, Alexander GL, Balm $\mathrm{RK}$, et al. Acute major gastrointestinal hemorrhage in inflammatory bowel disease. Gastrointest Endosc. 1999:49:153-7.

86. Robert JR, Sachar DB, Greenstein AJ. Severe gastrointestinal hemorrhage in Crohn's disease. Ann Surg. 1991;213(3):207-11.

87. Bini EJ, Weinshel EH, Falkenstein DB. Risk factors for recurrent bleeding and mortality in human immunodeficiency virus infected patients with acute lower Gl hemorrhage. Gastrointest Endosc. 1999;49:748-53.

88. Wong Kee Song LM, Baron TH. Endoscopic management of acute lower gastrointestinal bleeding. Am J Gastroenterol. 2008;103:1881-7.

89. Beejay U, Marcon NE. Endoscopic treatment of lower gastrointestinal bleeding. Curr Opin Gastroenterol. 2002;18:87-93.

90. Hirana H, Atsumi M, Sawai N, Ishimaru S. A case of ulcerative colitis with local bleeding treated by endoscopic injection of absolute ethanol and 1\% polidocanol. Gastroenterol Endosc. 1999;4:969-73.

91. Yoshida Y, Kawaguchi A, Mataki N, Matsuzaki K, Hokari R, Iwai A, et al. Endoscopic treatment of massive lower Gl hemorrhage in two patients with ulcerative colitis. Gastrointest Endosc. 2001;54:779-81.

92. Chen YI, Barkun A, Nolan S. Hemostatic powder TC-325 in the management of upper and lower gastrointestinal bleeding a two year experiences at a single institution. Endoscopy. 2015:47:167-71.

93. Smith SR, Murray D, Pockney PG, Bendinelli C, Draganic BD, Carroll R. Tranexamic acid for lower Gl hemorrhage: A randomized placebo-controlled clinical trial. Dis Colon Rectum. 2018:61:99-106.

94. Busch OR, van Delden OM, Gouma DJ. Therapeutic options for endoscopic hemostatic failures: the place of the surgeon and radiologist in gastrointestinal tract bleeding. Best Pract Res Clin Gastroenterol. 2008;22:341-54

95. Alonso-Coello P, Marzo-Castillejo M, Mascort JJ, Hervás AJ, Viña LM, Ferrús JA, et al. Guía de práctica clínica sobre el manejo del paciente con rectorragia. Gastroenterol Hepatol. 2002;25(10):605-32.

96. Daram SR, Lahr C, Tang SJ. Anorectal bleeding: etiology, evaluation and management. Gastrointest Endosc. 2012,76:406-17.

97. Pfenninger JL, Zainga GG. Common anorectal conditions. Part 1, Symptoms, and complaints. Am Fam Physician. 2001;63:2391-8.

98. Ellis DJ, Bevan PG. Proctoscopy and sigmoidoscopy. Br Med J. 1980,281:435-37.

99. Harper MB, Pope JB. Office Procedures-Flexible sigmoidoscopy. Prim care. 1997;24:341-57.

100. Ransohoff DF, Sander RS. Clinical Practice. Screening for colorecta cancer. N Engl J Med. 2002:346:40-4.

101. Iseli A. Sigmoidoscopy. Is it a general practice procedure? Aust Fam Physicians. 1999;28:61-4

102. Graham P. Sigmoidoscopy. Aust Fam Physicians. 1996;25:1403-4.

103. Winawer SJ, Miller C, Lightdale C, Herbert E, Ephram RC, Gordon L, et al. Patient response to sigmoidoscopy. Cancer. 1987;60:1905-8.

104. Lewis JD, Brown A, Localio AR, Schwartz JS. Initial evaluation of rectal bleeding in young persons: a cost-efectiveness analysis. Ann Inter Med. 2001;136:99-100.

105. Daram SR, Lahr C, Tang SJ. Anorectal bleeding: Etiology, evaluation, and management. Gastrointest Endosc. 2012;76:406-17.

106. Sato $Y$, Takayama T, Sagawa T, Hirakawa M, Ohnuma H, Miyanishi $K$ et al. Argon plasma coagulation treatment of hemorrhagic radiation proctopathy: The optimal settings for application and long-term outcome. Gastrointest Endosc. 2011;73:543-9.

107. Villavicencio RT, Rex DK, Rahmani E. Efficacy and complications of argon plasma coagulation for hematochezia related to radiation proctopathy. Gastrointest Endosc. 2002;55(1):70-4. 
108. Rustagi T, Corbett FS, Mashimo $\mathrm{H}$. Treatment of chronic radiation proctopathy with radiofrequency ablation. Gastrointest Endosc. 2015; 81:428-36.

109. Zhou C, Adler DC, Becker L, Chen Y, Tsai TH, Figueiredo M, et al. Effective treatment of chronic radiation proctitis using radiofrequency ablation. Therap Adv Gastroenterol. 2009;2:149-56.

110. Hou JK, Abudayyeh S, Shaib Y. Treatment of chronic radiation proctitis with cryoablation. Gastrointest Endosc. 2011;73:383-9.

111. Haas EM, Bailey HR, Farragher I. Application of 10 percent formalin for the treatment of radiation-induced hemorrhagic proctitis. Dis Colon Rectum. 2007; 50:213-7.

112. Pikarsky AJ, Belin B, Efron J, Weiss EG, Nogueras JJ, Wexner SD. Complications following formalin installation in the treatment of radiation induced proctitis. Int J Colorectal Dis. 2000;15:96-9.

113. Prasad V, Krishnamachari S. Chronic haemorrhagic radiation proctitis: A review. World J Gastointest Surg. 2016;267:483-91.

114. Brown SR. Haemorrhoids: an update on management. Ther Adv Chronic Dis. 2017:8:141-7.

115. Hall JF. Modern management of hemorrhoidal disease. Gastroenterol Clin N Am. 2013;42(4):759-72.

116. Lohsiriwat V. Treatment of hemorrhoids: A coloproctologist's view. World J Gastroenterol. 2015; 21(31):9245-52.

117. Davis BR, Lee-Kong SA, Migaly J, Feingold DL, Steele SR. The American Society of Colon and Rectal Surgeons Clinical Practice Guidelines for the Management of Hemorrhoids. Dis Colon Rectum. 2018;61:284-92.

118. Cocorullo G, Tutino R, Falco N, Licari L, Orlando G, Fontana T, et al. The non-surgical management for hemorrhoidal disease. A systematic review. G Chir. 2017;38(1):5-14

119. Andreia A. Rubber band ligation of hemorrhoids: A guide for complications. World J Gastrointest Surg. 2016;8:614-20.

120. MacRae HM, McLeod RS. Comparison of hemorrhoidal treatment modalities. A meta-analysis. Dis Colon Rectum. 1995;38:687-94.

121. Brown SR, Watson A. Comments to 'Rubber band ligation versus excisional haemorrhoidectomy for haemorrhoids'. Tech Coloproctol. 2016 20:659-61.

122. Yano T, Yano K. Comparison of injection sclerotherapy between $5 \%$ phenol in almond oil and aluminum potassium sulfate and tannic acid for grade 3 hemorrhoids. Ann Coloproctol. 2015;31:103-5.

123. Moser KH, Mosch C, Walgenbach M, Bussen DG, Kirsch J, Joos AK et al. Eficacy and safety of sclerotherapy with polidocanol foam in comparison with fluid sclerosant in the treatment of first grade haemorrhoidal disease: a randomised, controlled, single-blind, multicentre trial. Int J Colorectal Dis. 2013;28:1439-47.

124. Poen AC, Felt-Bersma RJ, Cuesta MA, Devillé W, Meuwissen SG. A randomized controlled trial of rubber band ligation versus infra-red coagulation in the treatment of internal haemorrhoids. Eur $\mathrm{J}$ Gastroenterol Hepatol. 2000;12(5):535-9

125. Marques CF, Nahas SC, Nahas CS, Sobrado CW Jr, Habr-Gama A Kiss DR. Early results of the treatment of internal hemorrhoid disease by infrared coagulation and elastic banding: a prospective randomized cross-over trial. Tech Coloproctol. 2006;10(4):312-7.

126. Felt-Bersma RJ, Tiersma ES, Cuesta MA. Rectal prolapse, rectal intussusception, rectocele, solitary rectal ulcer syndrome, and enterocele. Gastroenterol Clin North Am. 2008;37:645-68.

127. Cruveihier J. Anatomie pathologique du corps humain. Paris y Londres J.B. Bailliere; 1829

128. Martin CJ, Parks TG, Biggart JD. Solitary rectal ulcer syndrome in Northern Ireland. 1971-1980. Br J Surg. 1981;68:744-7.

129. Chiang JM, Changchien CR, Chen JR. Solitary rectal ulcer syndrome: an endoscopic and histological presentation and literature review. Int J Colorectal Dis. 2006;21:348-56.

130. Haray PN, Morris-Stiff GJ, Foster ME. Solitary rectal ulcer syndrome-an underdiagnosed condition. Int J Colorectal Dis. 1997:12:313-5.

131. Levine DS, Surawicz CM, Ajer TN, Dean PJ, Rubin CE. Diffuse excess mucosal collagen in rectal biopsies facilitates differential diagnosis of solitary rectal ulcer syndrome from other inflammatory bowel diseases. Dig Dis Sci. 1988;33:1345-52.

132. Ignjatovic A, Saunders BP, Harbin L, Clark S. Solitary 'rectal' ulcer syndrome in the sigmoid colon. Colorectal Dis. 2010;12:1163-4.

133. Endoscopic management of colonic diverticular bleeding. Digestive Endoscopy $2015 ; 27: 721-6$

134. McGuire $\mathrm{HH}$. Bleeding colonic diverticula - A reappraisal of natural-history and management. Ann Surg. 1994; 220:653-6.

135. Kaltenbach T, Watson R, Shah J, Friedland S, Sato T, Shergill A, et al. Colonoscopy with clipping is useful in the diagnosis and treatment of diverticular bleeding. Clin Gastroenterol Hepatol. 2012;10:131-7.

136. Yen EF, Ladabaum U, Muthusamy VR, Cello JP, McQuaid KR, Shah JN. Colonoscopic treatment of acute diverticular hemorrhage using endoclips. Dig Dis Sci. 2008;53:2480-5

137. Ishii N, Setoyama T, Deshpande GA, Omata F, Matsuda M, Suzuki S, et al. Endoscopic band ligation for colonic diverticular hemorrhage. Gastrointest Endosc. 2012;75:382-7
138. Bertoni G, Conigliaro R, Ricci E, Mortilla MG, Bedogni G, Fornaciari G. Endoscopic injection hemostasis of colonic diverticular bleeding: a case report. Endoscopy. 1990;22:154-5.

139. Prakash C, Chokshi H, Walden DT, Aliperti G. Endoscopic hemostasis in acute diverticular bleeding. Endoscopy. 1999;31:460-3.

140. Bloomfeld RS, Rockey DC, Shetzline MA. Endoscopic therapy of acute diverticular hemorrhage. Am J Gastroenterol. 2001;96:2367-72.

141. Ishii N, Hirata N, Omata F, Itoh T, Uemura M, Matsuda M, et al. Location in the ascending colon is a predictor of refractory colonic diverticular hemorrhage after endoscopic clipping. Gastrointest Endosc. 2012; 76:1175-81.

142. Witte JT. Band ligation for colonic bleeding: modification of multiband ligating devices for use with a colonoscope. Gastrointest Endosc. 2000;52:762-5.

143. Farrell JJ, Graeme-Cook F, Kelsey PB. Treatment of bleeding colonic diverticula by endoscopic band ligation: an in-vivo and ex-vivo pilot study. Endoscopy. 2003;35:823-9

144. Ishii N, Itoh T, Uemura M, Maruyama M, Horiki N, Setoyama T, et al. Endoscopic band ligation with a water-jet scope for the treatment of colonic diverticular hemorrhage. Dig Endosc. 2010;22:232-5.

145. Kanwal F, Dulai G, Jensen DM, Gralnek IM, Kovacs TOG, Machicado GA, et al. Major stigmata of recent hemorrhage on rectal ulcers in patients with severe hematochezia: endoscopic diagnosis, treatment, and outcomes. Gastrointest Endosc. 2003;57(4):462-8.

146. Chen-Kuan L, Cheng-Chao L, Hou-Tai C, Fang-Ming H, Tzong-Hsi L. Acute hemorrhagic rectal ulcer: An important cause of lower gastrointestinal bleeding in the critically ill patients. Dig Dis Sci. 2011;12:3631-7.

147. Al Khalloufi K, Laiyemo AO. Management of rectal varices in portal hypertension. World J Hepatol. 2015;7(30):2992-8.

148. Nojkov B, Cappell MS. Gastrointestinal bleeding from Diulafoy's lesión: Clinical presentation, endoscopic findings, and endoscopic therapy. World J Gastrointest Endosc. 2015;7(4):295-307.

149. Zuckerman GR, Prakash C. Acute lower intestinal bleeding: part I: clinical presentation and diagnosis. Gastrointest Endosc. 1998;48:606-17.

150. Leung Ki EL, Lau JY. New endoscopic hemostasis methods. Clin Endosc. 2012;45:224-9.

151. Guo SB, Gong AX, Leng J, Ma J, Ge LM. Application of endoscopic hemoclips for nonvariceal bleeding in the upper gastrointestinal tract. World J Gastroenterol. 2009;15:4322-6.

152. Setoyama $T$, Ishii N, Fujita $Y$. Endoscopic band ligation (EBL) is superio to endoscopic clipping for the treatment of colonic diverticular hemorrhage. Surg Endosc. 2011;25:3574-8.

153. Shibata S, Shigeno T, Fujimori K, Kanai K, Yoshizawa K Colonic diverticular hemorrhage: the hood method for detecting responsible diverticula and endoscopic band ligation for hemostasis. Endoscopy. 2014;46:66-9.

154. Campo R, Brullet E, Montané JM, Bombardó J, Sanfeliu J. Elastic band ligation in the bowel: is it really safe? Gastrointest Endosc. 1998;47:105-6.

155. Richter JM, Christensen MR, Colditz GA, Nishioka NS. Angiodysplasia. Natural history and efficacy of therapeutic interventions. Dig Dis Sci. 1989;34:1542-6.

156. Sharma R, Gorbien MJ. Angiodysplasia and lower gastrointestinal tract bleeding in elderly patients. Arch Intern Med. 1995;155:807-12.

157. Yeoh E, Tam W, Schoeman M, Moore J, Thomas M, Botten R, et al. Argon plasma coagulation therapy versus topical formalin for intractable rectal bleeding and anorectal dysfunction after radiation therapy for prostate carcinoma. Int J Radiat Oncol Biol Phys. 2013;87:954-9.

158. Silva RA, Correia AJ, Dias LM, Viana HL, Viana RL. Argon plasma coagulation therapy for hemorrhagic radiation proctosigmoiditis. Gastrointest Endosc. 1999;50:221-4.

159. Kwan V, Bourke MJ, Williams SJ, Gillespie PE, Murray MA, Kaffes AJ, et al. Argon plasma coagulation in the management of symptomatic gastrointestinal vascular lesions: experience in 100 consecutive patients with long-term follow-up. Am J Gastroenterol. 2006;101:58-63.

160. Olmos JA, Marcolongo M, Pogorelsky V, Herrera L, Tobal F, Dávolos JR. Longterm outcome of argon plasma ablation therapy for bleeding in 100 consecutive patients with colonic angiodysplasia. Dis Colon Rectum. 2006;49:1507-16.

161. Jayaraman V, Hammerle C, Lo SK, Jamil L, Gupta K. Clinical application and outcomes of over the scope clip device: initial US experience in humans. Diagn Ther Endosc. 2013:381873.

162. Anthony T, Penta P, Todd RD, Sarosi GA, Nwariaku F, Rege RV. Rebleeding and survival after acute lower gastrointestinal bleeding. Am J Surg. 2004; $188: 485-90$

163. Aytac E, Stocchi L, Gorgun E, Ozuner G. Risk of recurrence and longterm outcomes aft er colonic diverticular bleeding. Int $\mathrm{J}$ Colorectal Dis. 2014:29:373-8.

164. Swanson E, Mahgoub A, MacDonald R, Shaukat A. Medical and endoscopic therapies for angiodysplasia and gastric antral vascular ectasia: a systematic review. Clin Gastroenterol Hepatol. 2014;12:571-82.

165. Laine L, Shah A. Randomized trial of urgent vs. Elective colonoscopy in patients hospitalized with lower GI bleeding. Am J Gastroenterol. 2010;105:2636-41.

166. Mourad FH, Leong RW. Role of hemostatic powders in the managemen of lower gastrointestinal bleeding: A review. J Gastroenterol Hepatol. 2018;33(8):1445-53. 
167. Prei JC, Barmeyer C, Bürgel N, Daum S, Epple HJ, Günther U, et al. EndoClot polysaccharide hemostatic system in nonvariceal gastrointestinal bleeding: Results of a prospective multicenter observational pilot study. J Clin Gastroenterol. 2016;50:e95-100.

168. Chen YI, Barkun AN. Hemostatic powders in gastrointestinal bleeding: A systematic review. Gastrointest Endosc Clin N Am. 2015;25:535-52.

169. Clerc D, Grass F, Schäfer M, Denys A, Demartines N, Hübner M. Lower gastrointestinal bleeding-computed tomographic angiography, colonoscopy or both? World J Emerg Surg. 2017;12:1.

170. Jiang Z, Nimura $Y$, Hayashi $Y$, Kitasaka T, Misawa K, Fujiwara M, et al Lower gastrointestinal bleeding: predictive factors and clinical outcome for the patients who needed first-time mesenteric conventional angiography. Diagn Interv Radiol. 2018;24:23-7.

171. Sildiro-lu O, Muasher J, Bloom TA, Kapucu 囚, Arslan B, Angle JF, et al. Acute lower gastrointestinal bleeding: predictive factors and clinical outcome for the patients who needed first-time mesenteric conventional angiography. Diagn Interv Radiol. 2018;24:23-7.
172. d'Othée BJ, Surapaneni P, Rabkin D, Nasser I, Clouse M. Microcoil embolization for acute lower gastrointestinal bleeding. Cardiovasc Intervent Radiol. 2006;29(1):49-58.

173. Tan KK, Nallathamby V, Wong D, Sim R. Can superselective embolization be definitive for colonic diverticular hemorrhage? An institution's experience over 9 years. J Gastrointest Surg. 2010;14:112-8.

174. Urbano J, Manuel Cabrera J, Franco A, Alonso-Burgos A. Selective arterial embolization with ethylene - vinyl alcohol copolymer for control of massive lower gastrointestinal bleeding: feasibility and initial experience. J Vasc Interv Radiol. 2014;25(6):839-46.

175. Raohaeli T, Menon R. Current treatment of lower gastrointestinal hemorrhage. Clin Colon Rectal Surg. 2012;25:219-27.

176. Oakland K, Guyr R, Uberoy R, Hogg R, Mortensen N, Murphy MF, et al. Acute lower Gl bleeding in the UK: patients characteristics interventions and outcomes in the first nationwide audit. Gut. 2018; 67(4):654-62 